See discussions, stats, and author profiles for this publication at: https://www.researchgate.net/publication/342134771

\title{
Fokker-Planck-Poisson kinetics: Multi-phase flow beyond equilibrium
}

Preprint · June 2020

DOI: $10.13140 / R G .2 \cdot 2 \cdot 15446.88640$

CITATIONS

0

3 authors:

A Mohsen Sadr

RWTH Aachen University

7 PUBLICATIONS 17 CITATIONS

SEE PROFILE

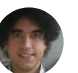

Hossein Gorji

École Polytechnique Fédérale de Lausanne

31 PUBLICATIONS 306 CITATIONS

SEE PROFILE

Some of the authors of this publication are also working on these related projects:

Project PICLas View project

Project Fokker-Planck Model for Multiphase Flows View project
READS

45

Marcel Pfeiffer

Universität Stuttgart

43 PUBLICATIONS 239 CITATIONS

SEE PROFILE 


\section{Fokker-Planck-Poisson kinetics: Multi-phase flow beyond equilibrium}

\begin{tabular}{|r|l|}
\hline Journal: & Journal of Fluid Mechanics \\
\hline Manuscript ID & Draft \\
\hline mss type: & JFM Papers \\
\hline Author: & n/a \\
\hline Keyword: List of Authors: & $\begin{array}{l}\text { Sadr, Mohsen; RWTH University Aachen Mathematics CCES } \\
\text { Pfeiffer, Marcel; Universitat Stuttgart, Institute of Space Systems } \\
\text { Gorji, Hossein; Ecole Polytechnique Federale de Lausanne, MCSS; } \\
\text { Stanford University, Energy Resource Engineering }\end{array}$ \\
\hline $\begin{array}{l}\text { Kinetic theory < Rarefied Gas Flow, Multiphase flow < Multiphase and } \\
\text { Particle-laden Flows, Non-continuum effects < Micro-/Nano-fluid } \\
\text { dynamics }\end{array}$ \\
\hline $\begin{array}{l}\text { Multi-phase phenomena remain at the heart of many challenging fluid } \\
\text { dynamics problems. Molecular fluxes at the interface determine the fate } \\
\text { of neighboring phases, yet their closure far from the continuum needs to } \\
\text { be modeled. Along the hierarchy of kinetic approaches, a multi-phase } \\
\text { particle method is devised in this study. Molecular interactions are } \\
\text { expressed via stochastic forces driven by the white noise, coupled to the } \\
\text { long-range attractions. The former is local and pursues diffusive } \\
\text { approximation of molecular collisions, whereas the latter takes a global } \\
\text { feature owing to mean-field forces. The obtained Fokker-Planck-Poisson } \\
\text { combination provides an efficient work-flow for physics-driven } \\
\text { simulations suitable for multi-phase phenomena far from the equilibrium. } \\
\text { Besides highlighting the computational efficiency of the method, various } \\
\text { archetypical and complex problems ranging from inverted-temperature- } \\
\text { gradient between droplets to spinodal decomposition are explored. } \\
\text { Detailed discussions are provided on different characteristics of the } \\
\text { droplets dispersed in low/high density background gases; including the } \\
\text { departure of heat-fluxes from Fourier's law as well as droplets growth in } \\
\text { spinodal phases. }\end{array}$ \\
\hline Abstracts \\
\hline
\end{tabular}

\section{SCHOLARONE" Manuscripts}




\title{
Fokker-Planck-Poisson kinetics: Multi-phase flow beyond equilibrium
}

\author{
Mohsen Sadr $^{1} \dagger$, Marcel Pfeiffer ${ }^{2}$ and M. Hossein Gorji ${ }^{3}$ \\ ${ }^{1}$ Applied and Computational Mathematics, RWTH Aachen University, Schinkestrasse 2, \\ D-52062 Aachen, Germany \\ ${ }^{2}$ Institute of Space Systems, University of Stuttgart, Pfaffenwaldring 29, \\ D-70569 Stuttgart, Germany \\ ${ }^{3}$ MCSS, Ecole Polytechnique Fédérale de Lausanne (EPFL), \\ CH-1015 Lausanne, Switzerland
}

(Received xx; revised $\mathrm{xx}$; accepted $\mathrm{xx}$ )

Multi-phase phenomena remain at the heart of many challenging fluid dynamics problems. Molecular fluxes at the interface determine the fate of neighboring phases, yet their closure far from the continuum needs to be modeled. Along the hierarchy of kinetic approaches, a multi-phase particle method is devised in this study. Molecular interactions are expressed via stochastic forces driven by the white noise, coupled to the long-range attractions. The former is local and pursues diffusive approximation of molecular collisions, whereas the latter takes a global feature owing to mean-field forces. The obtained Fokker-Planck-Poisson combination provides an efficient work-flow for physics-driven simulations suitable for multi-phase phenomena far from the equilibrium. Besides highlighting the computational efficiency of the method, various archetypical and complex problems ranging from inverted-temperature-gradient between droplets to spinodal decomposition are explored. Detailed discussions are provided on different characteristics of the droplets dispersed in low/high density background gases; including the departure of heat-fluxes from Fourier's law as well as droplets growth in spinodal phases.

\section{Key words:}

\section{Introduction}

Conventional hydrodynamics can be extended in order to account for multi-phase phenomena. The Young-Laplace model for surface-tension besides the Maxwell equation for evaporation/condensation are typically employed. Along with Newton's viscosity law and Fourier's law of heat-fluxes, they form linear-response approximations for unclosed variables arising from conservation laws. It is well established that these closures become less reliable once strong departures from the equilibrium is observed, e.g. see (Wörner 2012; Schwarzkopf et al. 2011). In practice, this continuum breakdown of multi-phase flows arises in near vacuum conditions and/or miniaturized devices. Plume-droplet interaction in space thrusters (Benson et al. 2004), shale gas flows through ultratight porous media (Alharthy et al. 2013) and laser-droplet interaction in high-energy lithography (Hudgins et al. 2016), can be mentioned as few examples.

$\dagger$ Email address for correspondence: sadr@mathcces.rwth-aachen.de 
The problem of modeling surface and flux terms can be circumvented by resetting conservation laws in the kinetic theory framework. The latter describes the dynamics by means of the molecular velocity distribution which accounts for the probability that a particle has a certain velocity at a given point in the space-time coordinate. Accordingly, various macroscopic field quantities can be recovered by conditional expectation of molecular quantities. The Boltzmann equation provides an accurate description of the velocity distribution in the dilute gas limit, subject to the molecular chaos assumption (Bird 1994; Chapman \& Cowling 1970; Cercignani 1988b). By modifying the Boltzmann collision operator, the Enskog equation can be devised for dense gases (Enskog 1922; Van Beijeren \& Ernst 1973; Resibois 1978). This is achieved by including the volume exclusion in the collision operator and adjusting the collision frequency. As the attractive forces of the molecular potential is represented by the mean-field theory, the Enskog-Vlasov (EV) equation is obtained (Vlasov 1978). The EV equation can account for liquids, gases and multi-phase flows accurately (Karkheck \& Stell 1981; Grmela 1971). While the EV equation is adopted as the basis of this study, meso-scale models for multi-phase phenomena can be derived from other routes as well. For example, the Landau-Lifschitz fluctuating hydrodynamics offers a framework to introduce correlations among macroscropic fluxes and hence yields improvement over conventional hydrodynamics (Landau \& Lifshitz 1959). Following this idea, Smootheddissipative particle-hydrodynamics has been proposed for simulations of multi-phase flows beyond hydrodynamics (Espanol \& Revenga 2003; Liu et al. 2007).

Foremost, direct computation of the velocity distribution from kinetic equations should deal with the high-dimensionality of molecular degrees-of-freedom. Moreover, an accurate discretization of the collision operator has to honour collisional scales which yields stiffness issues near the equilibrium. Finally, long-range interactions described in the EV equation introduces yet another challenge for efficient computations. Spectral and discrete velocity methods have been pursued aiming at accurate solution of Boltzmann and Enskog equations (see e.g. Broadwell (1964); Gamba \& Tharkabhushanam (2009); $\mathrm{Wu}$ et al. $(2013,2015,2016))$. The problem of high-dimensionality of kinetic systems can be relaxed by approximating the velocity distribution via finite number of moments. These so-called moment methods which are applicable for flows not too far from the equilibrium, have been devised using Grad's ansatz, Chapman-Enskog expansion, Maximum-Entropy solution and quadrature methods (see e.g. Kremer \& Rosa Jr (1988); Torrilhon (2016); Struchtrup \& Frezzotti (2019); Wang et al. (2020); Heylmun et al. (2019)).

Alternatively, the curse of high-dimensionality can be avoided by evolving samples of the distribution according to the underlying stochastic process; at the cost of introducing statistical noise. Direct Simulation Monte-Carlo (DSMC) introduced by Bird (1963) is most popular particle algorithm for rarefied gas flow simulations. By adopting same principles, Montanero \& Santos (1997a), Alexander et al. (1995), Frezzotti (1997) and Frezzotti et al. (2019) extended DSMC algorithm for the dense flows. However as DSMC can successfully circumvent the high-dimensionality issue by resetting the kinetic system into the Monte-Carlo framework, it inherits stiffness of the collision operator near the equilibrium. This fundamental issue lies on the fact that the stochastic process resulting from the collision operator is not continuous in the velocity space. Thus time step sizes smaller than the mean-collision-time and spatial discretization smaller than the mean-free-path should be adopted. 
The high-computational cost associated with dense collisions, motivated Jenny et al. (2010) to introduce a particle approach based on the Fokker-Planck (FP) approximation of the Boltzmann equation. The main idea behind the FP model is to describe the jump process resulting from the collision operator by an equivalent drift-diffusion process. Hence the resulting stochastic paths become continuous and thus the discretization constraints become more relaxed in comparison to DSMC type approaches. Further improvements of the FP model led to continuous stochastic description of dense gases on the basis of the Enskog equation (Sadr \& Gorji 2017). The FP model for dense gases (DFP) is constructed such that relaxation rates of various production terms of the Enskog collision operator, as well as the collisional transfer of conserved quantities during the collision, are honoured.

At a different front, the computation of long-range interaction based on the meanfield limit can introduce further complexities due to long tail of the molecular potential. In contrast to kernel based estimation of long-range interactions (Frezzotti et al. 2005; Piechór 1994; Frezzotti et al. 2018), a global solution algorithm can be devised as shown by Sadr \& Gorji (2019). First the attractive part of molecular potential can be approximated accurately via Green's function associated with an elliptic partial differential equation (PDE). The resulting screened-Poisson equation (SP) then can be adopted in order to approximate the Vlasov integral. Unlike density expansion methods which are local and require high regularity of the density field (He \& Doolen 2002; Korteweg 1901), the solution offered by the SP model retains global features of the Vlasov integral and allows for strong variations of the density field.

In this study, a solution algorithm is devised, which combines an accurate continuous description of the molecular collisions along with an efficient estimation of long-range interactions. The former is achieved by the DFP approximation of the Enskog equation, whereas the latter follows the SP model for the Vlasov integral. First in $\S 2$, the kinetic theory as a mathematical model of multi-phase flows is described. In particular, the Enskog collision operator and the Vlasov integral are reviewed in $\S 2.1$ and $\S 2.2$, respectively. Then, the FP approximation of the Enskog equation along with the SP model of long-range forces are reviewed in $\S 3.1$ and $\S 3.2$, respectively. Next, an efficient particle Monte-Carlo solution algorithm for the Fokker-Planck-Poisson (DFP-SP) model suitable for multi-phase flows is assembled in $\S 4$.

The resulting DFP-SP model then is validated in $\S 5$, by studying evaporation of droplets in non-equilibrium settings. As the benchmark, numerical simulation based on the EV equation is obtained by combining the Enskog Simulation Monte-Carlo derived by Montanero \& Santos (1997b) for collisions, and Legendre-Gauss quadrature for the Vlasov integral. Next, the DFP-SP model is adopted to study inverted-temperaturegradients and spinodal decomposition problems in $\S 6$ and $\S 7$, respectively. For the former, the heat-transfer between two liquid slabs kept at a background gas is studied in a one-dimensional geometry. The results are compared with respect to benchmark EV solutions. By investigation of the single droplet evaporation, further insight is gained with respect to origin of inverted temperature gradient phenomenon. Finally gas-droplet decomposition in meta-stable setting known as the spinodal decomposition is studied in two and three dimensional settings. The simulation results are found to exhibit Lifschitz-Slyozov growth rate for the droplets. The validation study along with detailed simulation results provided for inverted temperature gradient and spinodal 
decomposition confirm efficiency and accuracy of the proposed DFP-SP model. The paper concludes in $\S 8$, where an outline of future improvements is discussed.

\section{Review of Enskog-Vlasov kinetic model}

As number of molecules which constitute a typical fluid portion is immense, it is more natural to apply a statistical approach if we are interested in macroscopic behaviour of the system. The kinetic theory provides a description of the flow by means of the molecular distribution. Consider an ensemble of random particles with translational degrees-offreedom. The state of the ensemble is fully determined by random variables $X(t) \in$ $\mathbb{R}^{3}$ and $V(t) \in \mathbb{R}^{3}$ which account for position and velocity of a sample at time $t \in$ $\mathbb{R}^{+}$, respectively. Equivalently, we can deduce the single-particle velocity distribution at position $x$ and velocity $v$ by

$$
\mathcal{F}(v, x, t)=\mathcal{M E}[\delta(x-X(t)) \delta(v-V(t))],
$$

where $\mathbb{E}[$.$] is the expectation, \delta($.$) denotes the Dirac delta and \mathcal{M}$ accounts for the total mass of the system (see e.g. Gorji (2014)). In absence of boundary effects, the distritbution $\mathcal{F}$ evolves due to the inter-molecular interactions. For our system of particles with molecular mass $m$, consider the Sutherland molecular potential

$$
\phi(r)= \begin{cases}+\infty & r<\sigma \\ \phi_{0}\left(\frac{\sigma}{r}\right)^{6} & r \geqslant \sigma\end{cases}
$$

where $r$ is the distance between any two particles, $\sigma$ indicates the effective diameter and $\phi_{0}$ is a scaling factor. In principle for a given inter-molecular potential, the Liouville equation offers evolution of the multi-particle distribution as shown by Cercignani (1988a). However if further simplifications are adopted i.e. the mean-field limit for long-range interactions along with the molecular chaos assumption, a closed form equation for $\mathcal{F}$ can be deduced (Grmela 1971; Karkheck \& Stell 1981; Cercignani 1988a). Contributions of short-range interactions to the evolution of $\mathcal{F}$ can be captured by considering an elastic collision operator, whereas the $N$-body problem arising from the long-tail interaction can be approximated by the mean-field limit (Vlasov 1978). Hence, the EV equation

$$
\frac{\partial \mathcal{F}}{\partial t}+\frac{\partial\left(\mathcal{F} v_{i}\right)}{\partial x_{i}}-\frac{1}{m} H_{i} \frac{\partial \mathcal{F}}{\partial v_{i}}=S^{\text {Ensk }}(\mathcal{F})
$$

can be derived where the Enskog collision operator $S^{\text {Ensk }}(\mathcal{F})$ accounts for the contribution of molecular collisions. Furthermore, the conservative force field $H_{i}:=\partial \Phi / \partial x_{i}$ accounts for long-range attraction and follows the gradient of the mean-field potential $\Phi$. Here and henceforth the Einstein summation convention is used to economize our notation.

In follow-up subsections, first we review the Enskog collision operator and next a short description on the mean field potential $\Phi$ is provided.

\subsection{Enskog operator}

The short-range interactions have two main influences on evolution of the velocity distribution: relaxation towards equilibrium and transport of conserved quantities. Note that the latter is only the case for dense flows. The Enskog collision operator derived by Enskog (1922) and then modified by Van Beijeren \& Ernst (1973); Resibois (1978), is an extension of the Boltzmann collision operator by including the volume of particles. The 
standard Enskog collision operator for hard-sphere collisions reads

$$
\begin{aligned}
S^{\text {Ensk }}(\mathcal{F})=\frac{1}{m} & \int_{\mathbb{R}^{3}} \int_{0}^{2 \pi} \int_{0}^{\sigma}\left[Y\left(x+\frac{1}{2} \sigma \hat{k}\right) \mathcal{F}\left(v^{*}, x\right) \mathcal{F}\left(v_{1}^{*}, x+\sigma \hat{k}\right)\right. \\
& \left.-Y\left(x-\frac{1}{2} \sigma \hat{k}\right) \mathcal{F}(v, x) \mathcal{F}\left(v_{1}, x-\sigma \hat{k}\right)\right] g \mathcal{H}\left(g_{i} \hat{k}_{i}\right) \hat{b} d \hat{b} d \hat{\epsilon} d v_{1}
\end{aligned}
$$

where $\hat{k}$ indicates unit vector connecting the center of a particle to its colliding pair with subscript $(.)_{1}, \mathcal{H}($.$) the Heaviside step function, g$ is the relative velocity of colliding pair, and $\hat{b}$ and $\hat{\epsilon}$ are the impact parameter and scattering angle, respectively. Note that in case of hard-sphere potential, the impact parameter relates to deflection angle $\chi$ via $\hat{b}=\sigma \cos (\chi / 2)$ where $\chi \in[0, \pi)$. For further details of the Enskog and Boltzmann collision operators see e.g., Chapman \& Cowling (1970), Hirschfelder et al. (1964) and Bird (1994).

In comparison to the dilute limit, the Enskog collision operator admits a diameter distance between the colliding pair as well as an increase in collision rate by $Y$. The standard Enskog equation estimates the pair correlation factor $Y$ to be a function of density at the contact of colliding spheres, which can be chosen such that the correct equilibrium pressure is guaranteed. By means of the Carnahan-Starling equation of state, we get

$$
\begin{aligned}
Y & =\frac{1}{2} \frac{2-\eta}{(1-\eta)^{3}} \\
\text { with } \quad \eta & :=n b / 4,
\end{aligned}
$$

where $b:=2 \pi \sigma^{3} / 3$ is the second virial coefficient (Carnahan \& Starling 1969). Furthermore, the number density is denoted by $n$ which gives the density $\rho=m n$.

In addition to the curse of dimensionality associated with $\mathcal{F}$, the increase of collision rate along with the non-locality of collision operator lead to further computational complications. Although direct Monte-Carlo particle methods can cope with the highdimensionality at the cost of introducing statistical noise, they inherit the issues with non-locality of collision step along with resolving collisional scales.

\subsection{Vlasov integral}

By applying the mean-field limit, the attractive force of Eq. (2.3) is represented as the derivative of the Vlasov integral

$$
\Phi(x, t)=\int_{r>\sigma} \phi(r) n(y, t) d y,
$$

where $r:=|y-x|$ and $\mid$. | denotes Euclidean distance. Although the Vlasov integral can be computed using quadrature rules as discussed in Appendix A.2, long-tails and mesh-refinement near $r=\sigma$ make the numerical integration expensive in practice.

\section{Fokker-Planck-Poisson Model}

Here a detailed description of the Fokker-Planck-Poisson model covering both short- and long-range interactions is discussed. First in $\S 3.1$, the FP approximation of collisions in dense gases is reviewed. Next, we explain the elliptic model that captures the long-range forces globally, by means of the SP equation in $\S 3.2$. 


\subsection{Short-range interactions}

The computational cost associated with resolving the Enskog collision operator i.e. right hand side (rhs) of Eq. (2.3), can be decoupled from collisional scales once the underlying jump process is described by an equivalent continuous process. Furthermore, if the stochastic paths generated by the latter process depends only on local moments of the distribution, efficient cell-based parallelization can be exploited.

Let us consider an Itô drift-diffusion process as a general prototype of such continuous process. Then, the task becomes to find a drift-diffusion closure such that the resulting single-particle velocity distribution evolves consistent with the underlying jump process, up to a desired degree of moments. From physical point of view such drift-diffusion process expresses all the forces acted on a sample particle into a portion which is correlated to the particle's state and a remainder being purely random. The former is governed by the drift whereas the latter is controlled by the diffusion coefficient.

Consider position and velocity of particles given by random variables $(X(t), V(t))$. Suppose that evolution of particles can be modeled through stochastic differential equations (SDEs) with drift $A$ and diffusion $D$ in velocity space, as well as a spatial drift $\hat{A}$ in the physical space

$$
\left\{\begin{array}{l}
d V_{i}=A_{i} d t+D d W_{t, i} \text { and } \\
d X_{i}=V_{i} d t+\hat{A}_{i} d t
\end{array}\right.
$$

where $i \in\{1,2,3\}$. Here $d W_{t, i}:=W_{i}(t+d t)-W_{i}(t)$ indicates the increment of the Wiener process for the $i$ th component of particle velocity which follows a Gaussian distribution with zero mean and variance of $d t$, i.e. $d W_{t, i} \sim \mathcal{N}(0, d t)$. Using Itô's lemma, evolution of the corresponding $\mathcal{F}$ follow a $\mathrm{FP}$ equation of the form

$$
\frac{\partial \mathcal{F}}{\partial t}+\frac{\partial\left(\mathcal{F} v_{i}\right)}{\partial x_{i}}=S^{\mathrm{DFP}}(\mathcal{F})
$$

where

$$
S^{\mathrm{DFP}}(\mathcal{F})=\underbrace{-\frac{\partial\left(\mathcal{F} A_{i}\right)}{\partial v_{i}}+\frac{1}{2} \frac{\partial^{2}\left(D^{2} \mathcal{F}\right)}{\partial v_{i} \partial v_{i}}}_{\text {relaxation rates }} \underbrace{-\frac{\partial\left(\mathcal{F} \hat{A}_{i}\right)}{\partial x_{i}}}_{\text {col. transfer }} .
$$

For relationship between the FP equation and SDEs see e.g. Oksendal (2013). The drift $A$ and diffusion $D$ allow us to fix the rate at which non-conservative velocity moments including kinetic stress-tensor

$$
\pi_{i j}^{\mathrm{kin}}=\int_{\mathbb{R}^{3}} v_{\langle i}^{\prime} v_{j\rangle}^{\prime} \mathcal{F} d v
$$

and kinetic heat-flux vector

$$
q_{i}^{\mathrm{kin}}=\frac{1}{2} \int_{\mathbb{R}^{3}} v_{i}^{\prime} v_{j}^{\prime} v_{j}^{\prime} \mathcal{F} d v,
$$

relax due to collisions. Note that subscript $\langle$.$\rangle denotes the deviatoric part of the tensor$ and $v^{\prime}:=v-U$ is the fluctuating velocity with $U=(1 / \rho) \int v \mathcal{F} d v$ being the bulk velocity.

The sparial drift $\hat{A}$ accounts for the collisional transfer resulting from dense effects. From physical point of view, the introduced drift in the position-update corrects the particle position due to the volume exclusion. In other words, in absence of such correction the 
described SDEs only provide velocity and position of particles with vanishing diameter.

By considering the spatially homogeneous setting, we fix $A$ and $D$ such that the resulting moment system remain consistent with the Enskog equation up to the heat-fluxes. Accordingly, the following constraints

$$
\begin{aligned}
& \int_{\mathbb{R}^{3}} v_{\langle i}^{\prime} v_{j\rangle}^{\prime} S^{\mathrm{DFP}}(\mathcal{F}) d v=\int_{\mathbb{R}^{3}} v_{\langle i}^{\prime} v_{j\rangle}^{\prime} S^{\text {Ensk }}(\mathcal{F}) d v \quad \text { and } \\
& \int_{\mathbb{R}^{3}} v_{i}^{\prime} v_{j}^{\prime} v_{j}^{\prime} S^{\mathrm{DFP}}(\mathcal{F}) d v=\int_{\mathbb{R}^{3}} v_{i}^{\prime} v_{j}^{\prime} v_{j}^{\prime} S^{\mathrm{Ensk}}(\mathcal{F}) d v
\end{aligned}
$$

are devised. In order to obtain a tractable expression for the rhs of Eq. (3.6)-(3.7), here we approximate moments of the Enskog collision operator by applying Maxwell molecules. Therefore we get

$$
\begin{aligned}
& \int_{\mathbb{R}^{3}}\left(A_{i} v_{j}^{\prime}+A_{j} v_{i}^{\prime}-\frac{2}{3} A_{k} v_{k}^{\prime} \delta_{i j}+\frac{2}{3} \delta_{i j} D^{2}\right) \mathcal{F} d v=-Y \frac{p^{\mathrm{kin}}}{\mu^{\mathrm{kin}}} \pi_{i j}^{\mathrm{kin}} \text { and } \\
& \int_{\mathbb{R}^{3}}\left(A_{i} v_{j}^{\prime} v_{j}^{\prime}+2 A_{j} v_{j}^{\prime} v_{i}^{\prime}\right) \mathcal{F} d v=-Y \frac{2}{3} \frac{p^{\mathrm{kin}}}{\mu^{\mathrm{kin}}} q_{i}^{\mathrm{kin}} .
\end{aligned}
$$

Note that here kinetic ideal gas viscosity coefficient is denoted by $\mu^{\text {kin }}$, ideal gas equilibrium pressure is $p^{\mathrm{kin}}=n k_{b} T$ and superscript (. $)^{\mathrm{kin}}$ indicates kinetic contribution.

Next, the extra transport resulting from spatial dependencies of the Enskog operator is accounted by the spatial drift. The degrees of freedom in extra streaming induced by $\hat{A}$ are set such that the resulting FP model remain consistent with the Enskog collision operator up to the heat-fluxes. Let $\psi=\left[1, v_{i}, v_{j} v_{j} / 2\right]^{T}$, by the Taylor expansion of $\mathcal{F}(x \pm \sigma \hat{k})$ and $Y(x \pm \sigma \hat{k} / 2)$ around $x$, the conservative moments of Enskog collision operator can be formulated as fluxes

$$
\begin{aligned}
& \int_{\mathbb{R}^{3}} \psi_{l} S^{\text {Ensk }}(\mathcal{F}) d v=-\frac{\partial \Psi_{l k}^{\phi}}{\partial x_{k}}, \text { where } \\
& \Psi_{l k}^{\phi}=\frac{Y \sigma}{2 m} \iiint \int\left(\psi_{l}^{*}-\psi_{l}\right) \mathcal{F F}_{1} \hat{k}_{k} g \hat{b} d \hat{b} d \hat{\epsilon} d v_{1} d v \\
& \quad+\frac{Y \sigma^{2}}{4 m} \iiint\left(\psi_{l}^{*}-\psi_{l}\right) \hat{k}_{k} \hat{k}_{j} \mathcal{F F}_{1} \frac{\partial}{\partial x_{j}} \ln \left(\frac{\mathcal{F}}{\mathcal{F}_{1}}\right) g \hat{b} d \hat{b} d \hat{\epsilon} d v_{1} d v \\
& \quad+\cdots
\end{aligned}
$$

as shown by Chapman \& Cowling (1970). In particular, the corrections accounted by the spatial drift $\hat{A}$ become

$$
\begin{gathered}
\frac{\partial}{\partial x_{k}} \int_{\mathbb{R}^{3}} \hat{A}_{k} \psi_{l} \mathcal{F} d v \approx \frac{\partial \Psi_{l k}^{\phi}}{\partial x_{k}} \quad \text { for } l=1, \ldots, \operatorname{dim}(\psi) . \\
\text { Cambridge University Press }
\end{gathered}
$$


Assuming Maxwellian for $\mathcal{F}$ in $\partial_{x_{j}} \ln \left(\mathcal{F} / \mathcal{F}_{1}\right)$ inside the second integral of Eq. (3.11) and ignoring higher order terms, a closure for collisional transfer can be derived

$$
\begin{aligned}
& \int_{\mathbb{R}^{3}} \hat{A}_{i} \mathcal{F} d v=0 \\
& \int_{\mathbb{R}^{3}} \hat{A}_{i} v_{j} \mathcal{F} d v=n b Y\left(p^{\mathrm{kin}} \delta_{i j}+2 / 5 \pi_{i j}^{\mathrm{kin}}\right)-w\left(\frac{\partial U_{k}}{\partial x_{k}} \delta_{i j}+\frac{5}{6} \frac{\partial U_{\langle i}}{\partial x_{j\rangle}}\right) \\
\text { and } & \frac{1}{2} \int_{\mathbb{R}^{3}} \hat{A}_{i} v_{j} v_{j} \mathcal{F} d v=\frac{3}{5} n b Y q_{i}^{\mathrm{kin}}-c_{v} w \frac{\partial T}{\partial x_{i}} .
\end{aligned}
$$

Here $c_{v}=3 k_{b} /(2 m)$ is the heat capacity at constant volume,

$$
w=(n b)^{2} Y \sqrt{m k_{b} T} /\left(\pi^{3 / 2} \sigma^{2}\right)
$$

is the second viscosity coefficient, $T=\int_{\mathbb{R}^{3}} v_{j}^{\prime} v_{j}^{\prime} \mathcal{F} d v /\left(3 n k_{b}\right)$ is the temperature and $k_{b}$ denotes the Boltzmann constant.

A simple set of polynomial ansatz to fulfill Eqs. (3.8)-(3.9) in combination with Eqs. (3.13)-(3.15) is adopted. Following Sadr \& Gorji (2017), the cubic FP model for dense collisions

$$
\begin{aligned}
A_{i} & =c_{i j} v_{j}^{\prime}+\gamma_{i}\left(v_{j}^{\prime} v_{j}^{\prime}-\frac{3 k_{b} T}{m}\right)+\Lambda\left(v_{i}^{\prime} v_{j}^{\prime} v_{j}^{\prime}-\frac{2 q_{i}^{\mathrm{kin}}}{\rho}\right), \\
D & =\sqrt{\frac{k_{b} T}{\tau m}} \\
\text { and } \quad \hat{A}_{i} & =\hat{c}_{i j} v_{j}^{\prime}+\hat{\gamma}_{i}\left(v_{j}^{\prime} v_{j}^{\prime}-\frac{3 k_{b} T}{m}\right)+\hat{\Lambda}\left(v_{i}^{\prime} v_{j}^{\prime} v_{j}^{\prime}-\frac{2 q^{\mathrm{kin}}}{\rho}\right)
\end{aligned}
$$

is devised. The time scale $\tau$ is set using kinetic scales $\tau=2 \mu^{\text {kin }} /\left(p^{\text {kin }} Y\right)$. The coefficients of the tensors $c$ and $\hat{c}$ along with the vectors $\gamma$ and $\hat{\gamma}$ need to be computed based on velocity moments. The cubic term is responsible to make sure that the SDEs remain stable (see (Risken 1989; Gorji et al. 2011)). The coefficients of the cubic terms are set to

$$
\begin{aligned}
& \Lambda=-\frac{\left|\operatorname{det}\left(u_{i j}\right)\right|}{\left(u^{(2)}\right)^{4} \tau} \text { and } \\
& \hat{\Lambda}=-\epsilon \frac{n b Y}{k_{b} T / m},
\end{aligned}
$$

where $\epsilon=10^{-3}$, $\operatorname{det}($.$) indicates the determinant and$

$$
u_{i_{1} \ldots i_{n}}^{(k)}:=\frac{1}{\rho} \int_{\mathbb{R}^{3}}\left|v^{\prime}\right|^{k} v_{i_{1}}^{\prime} v_{i_{2}}^{\prime} \ldots v_{i_{n}}^{\prime} \mathcal{F} d v ; \quad i_{k} \in\{1,2,3\}
$$

By substituting the ansatz for $A$ and $D$ in equations for the relaxation rates Eqs. (3.8)(3.9), one can show that

$$
\begin{gathered}
c_{i k} u_{k j}^{(0)}+c_{j k} u_{k i}^{(0)}+\gamma_{i} u_{j}^{(2)}+\gamma_{j} u_{i}^{(2)}=-2 \Lambda u_{i j}^{(2)} \quad \text { and } \\
c_{i j} u_{j}^{(2)}+2 c_{j k} u_{i j k}^{(0)}+\gamma_{i}\left(u^{(4)}-\left(u^{(2)}\right)^{2}\right)+2 \gamma_{j}\left(u_{i j}^{(2)}-u^{(2)} u_{i j}\right) \\
=-\Lambda\left(3 u_{i}^{(4)}-u_{i}^{(2)} u^{(2)}-2 u_{j}^{(2)} u_{i j}^{(0)}\right)+\frac{5}{6} \frac{Y p^{\mathrm{kin}}}{\mu^{\mathrm{kin}}} q_{i}^{\mathrm{kin}} .
\end{gathered}
$$


Equivalently for $\hat{A}$ we get

$$
\begin{aligned}
& \hat{c}_{j k} \pi_{i k}^{\mathrm{kin}}+\hat{c}_{j i} p^{\mathrm{kin}}+2 \hat{\gamma}_{j} q_{i}^{\mathrm{kin}}=-\rho \hat{\Lambda} u_{i j}^{(2)} \\
&+n b Y\left(p^{\mathrm{kin}} \delta_{i j}+2 / 5 \pi_{i j}^{\mathrm{kin}}\right)-w\left(\frac{\partial U_{k}}{\partial x_{k}} \delta_{i j}+\frac{5}{6} \frac{\partial U_{\langle i}}{\partial x_{j\rangle}}\right) \text { and } \\
& \hat{c}_{i j} q_{j}^{\mathrm{kin}}+\frac{1}{2} \rho \hat{\gamma}_{i}\left(u^{(4)}-\left(u^{(2)}\right)^{2}\right)=\frac{3}{5} n b Y q_{i}^{\mathrm{kin}}-w c_{v} \frac{\partial T}{\partial x_{i}}-\frac{1}{2} \rho \hat{\Lambda}\left(u_{i}^{(4)}-u_{i}^{(2)} u^{(2)}\right),
\end{aligned}
$$

where $i, j, k \in\{1,2,3\}$. The unknowns $c, \gamma, \hat{c}$ and $\hat{\gamma}$, can be found by solving two linear systems

$$
A_{v}\left(\begin{array}{c}
c_{1,1} \\
\vdots \\
c_{3,3} \\
\gamma_{1} \\
\vdots \\
\gamma_{3}
\end{array}\right)=b_{v} \quad \text { and } \quad A_{x}\left(\begin{array}{c}
\hat{c}_{11} \\
\vdots \\
\hat{c}_{3,3} \\
\hat{\gamma}_{1} \\
\vdots \\
\hat{\gamma}_{3}
\end{array}\right)=b_{x}
$$

where the matrices $A_{v}$ and $A_{x}$ together with rhs matrices $b_{v}$ and $b_{x}$ are read from Eqs. (3.23)-(3.24) and Eqs. (3.25)-(3.26), respectively.

\subsection{Long-range interactions}

The contribution of the long-range molecular potential in the evolution of molecular velocity distribution function can be accurately accounted by the Vlasov mean-field limit. Although the direct numerical evaluation of long-ranged forces through the Vlasov integral is grid-independent however it can lead to high-computational cost due to relatively large support of the kernel. In practice, global solutions over a spatial discretization turn out to be less expensive (Sadr \& Gorji 2019). Consider an approximation $\tilde{\phi}(r)$ to the attractive part of molecular potential $\phi(r)$ where

$$
\begin{aligned}
\tilde{\phi}(r) & =a G(r) \quad \text { and } \\
G(r) & =\frac{e^{-\lambda r}}{4 \pi r} .
\end{aligned}
$$

An estimate of fitting parameters $a$ and $\lambda$ can be obtained by solving an optimization problem of the type

$$
(a, \lambda)=\underset{r \in(\sigma, \infty)}{\operatorname{argmin}}\left(\left\|\partial_{r} \phi(r)-\partial_{r} \tilde{\phi}(r)\right\|_{2}^{2}\right)
$$

for a given molecular potential. Here, $\|.\|_{2}$ indicates the $L^{2}$ norm. This ansatz is motivated by the fact that $G(r)$ is the Green function of the SP equation

$$
\left(\Delta-\lambda^{2}\right) \tilde{\Phi}_{r>0}(x, t)=n(x, t) ; \quad\left(\forall x \in \mathbb{R}^{3}\right),
$$

in three dimensional space. As shown above, the parameters $a$ and $\lambda$ give us flexibility to fit $\tilde{\phi}(r)$ to our desired potential. Hence, by substituting $\tilde{\phi}(r)$ in the Vlasov integral we 
obtain

$$
\begin{aligned}
\Phi(x, t) & =\int_{r>\sigma} \phi(r) n(y, t) d y \\
& \approx a \underbrace{\int_{r>0} G(r) n(y, t) d y}_{\tilde{\Phi}_{r>0}}-a \underbrace{\int_{r<\sigma} G(r) n(y, t) d y}_{\tilde{\Phi}_{r<\sigma}} \\
& =a\left(\tilde{\Phi}_{r>0}-\tilde{\Phi}_{r<\sigma}\right) .
\end{aligned}
$$

Further by assuming that the number density is smooth within $r \in(0, \sigma)$, an explicit expression for $\tilde{\Phi}_{r<\sigma}$ can be derived. In particular, using change of variables

$$
\begin{aligned}
\tilde{\Phi}_{r<\sigma}(x, t) & =\int_{r<\sigma} G(|y-x|) n(y, t) d^{3} y \\
& =\int_{|y|<\sigma} G(|y|) n(y-x, t) d^{3} y,
\end{aligned}
$$

and deploying Taylor's expansion for $n(y-x, t)$ around $(x, t)$, it can be shown that

$$
\begin{aligned}
\tilde{\Phi}_{r<\sigma}(x, t)= & n(x, t) \underbrace{\int_{|y|<\sigma} G(|y|) d^{3} y}_{\text {analytical expr. }}+\frac{1}{2} \frac{\partial^{2} n(x, t)}{\partial x_{j} \partial x_{j}} \underbrace{\int_{|y|<\sigma} G(|y|) y_{j} y_{j} d^{3} y}_{\text {analytical expr. }}+\ldots \\
\approx & n(x, t)\left[e^{-\lambda \sigma}\left(-\lambda \sigma+e^{\lambda \sigma}-1\right) / \lambda^{2}\right] \\
& +\frac{\partial^{2} n(x, t)}{\partial x_{j} \partial x_{j}}\left[e^{-\lambda \sigma}\left(-\lambda^{3} \sigma^{3} / 6-\lambda^{2} \sigma^{2} / 2-\lambda \sigma+e^{\lambda \sigma}-1\right) / \lambda^{4}\right]
\end{aligned}
$$

by second order truncation.

Therefore, instead of solving the Vlasov integral Eq. (2.7) directly, we find solution of the SP equation globally where the evaluated number density is fed as the source term. Fast Poisson solvers can be adjusted to the SP problem in the bounded domain $\Omega_{x} \subset \mathbb{R}^{3}$ with Dirichlet boundary conditions that provide the unique solution. The values of the potential on the boundaries of the domain $\partial \Omega_{x}$ are computed using the kernel $G(r)$ along with the boundary condition of $\mathcal{F}$. The detailed numerics of the SP model is presented $\S 4.2$.

\section{Particle method for Fokker-Planck-Poisson model}

Here we discuss a stochastic particle method for path simulations based on the devised FP approximation of the Enskog collision operator. Then, a discretization of the ScreenPoisson equation is provided. A solution algorithm combining both is outlined at the end.

\subsection{Stochastic particle method}

Since the SDEs (3.1a)-(3.1b) do not admit closed form time integration (due to nonlinearity of the drift), a numerical scheme has to be adopted. As explained by Jenny et al. (2010); Gorji et al. (2011); Gorji \& Jenny (2014), simple Euler-Maruyama scheme does not guarantee energy conservation on average. Given the fact that the Langevin 
equation with linear drift allows for explicit time integration, a decomposition of the SDEs into a linear drift portion and a remainder is pursued.

Following Sadr \& Gorji (2017), we consider a spatial discretization where each cell $c$ contains $N_{p,(c)}$ particles. Then the evolution of each particle is described via explicit time-stepping scheme, where the solution at time step $(n+1)$ is computed based on the observables estimated at $(n)$ th step.

Realizations of the SDEs (3.1a)-(3.1b), through particles with weights $w^{(k)}$, velocities $V^{(k)}$ and positions $X^{(k)}$ with $k \in\left\{1, \ldots, N_{p}\right\}$, provide an estimate

$$
\mathcal{F}(v, x, t) \approx \sum_{k=1}^{N_{p}} w^{(k)} \delta\left(V^{(k)}(t)-v\right) \delta\left(X^{(k)}(t)-x\right)
$$

of the distribution which can be used to obtain various observables. By generalizing particle discretization introduced by Gorji \& Jenny (2014), velocity and position of each particle get updated as

$$
\left\{\begin{array}{l}
V_{i}^{(*)}=\left\langle V_{i}\right\rangle_{(c)}^{(n)}+\alpha_{(c)} \tilde{V}_{i}^{(n)} \\
X_{i}^{(n+1)}=X_{i}^{(n)}+V_{i}^{(n+1)} \Delta t+\delta \tilde{X}_{i}^{(n)}
\end{array}\right.
$$

where

$$
\begin{aligned}
\tilde{V}_{i}^{(n)} & =V_{i}^{\prime,(n)} e^{-\Delta t Y^{(n)} / \tau^{(n)}}+c_{i j}^{(n)} V_{j}^{\prime,(n)}+\gamma_{i}^{(n)}\left(V_{j}^{\prime,(n)} V_{j}^{\prime,(n)}-3 k_{b} T^{(n)} / m\right) \\
& +\Lambda\left(V_{i}^{\prime,(n)} V_{j}^{\prime,(n)} V_{j}^{\prime,(n)}-2 q_{i}^{(n)} / \rho^{(n)}\right)+\sqrt{\frac{k T^{(n)} Y^{(n)}}{\tau^{(n)} m}\left(1-e^{\frac{-2 \Delta t Y^{(n)}}{\tau^{(n)}}}\right)} \xi_{i}
\end{aligned}
$$

and

$$
\begin{aligned}
\delta \tilde{X}_{i}^{(n)}=\left(\hat{c}_{i j}^{(n)} V_{j}^{\prime,(n)}\right. & +\hat{\gamma}_{i}^{(n)}\left(V_{j}^{\prime,(n)} V_{j}^{\prime,(n)}-3 k_{b} T^{(n)} / m\right) \\
& +\hat{\Lambda}^{(n)}\left(V_{i}^{\prime,(n)} V_{j}^{\prime,(n)} V_{j}^{\prime,(n)}-2 q_{i}^{(n)} / \rho^{(n)}\right) \Delta t .
\end{aligned}
$$

Notice that $V^{(*)}$ is modified accordingly once external or long-range forces are considered; otherwise we have $V^{(n+1)}=V^{(*)}$. Here $\xi_{i}$ is a random number drawn from the standard normal distribution, i.e., $\xi_{i} \sim \mathcal{N}(0,1)$, and $\alpha^{(c)}$ is the scaling factor which guarantees conservation of kinetic energy at each cell for a given time step

$$
\alpha_{(c)}=\frac{\left\langle V_{j}^{\prime,(n)} V_{j}^{\prime,(n)}\right\rangle_{(c)}}{\left\langle\tilde{V}_{j}^{(n)} \tilde{V}_{j}^{(n)}\right\rangle_{(c)}} .
$$

Note that $\langle.\rangle^{(c)}$ indicates a moment estimator. Using Eq. (4.1) we observe that

$$
\langle\hat{\phi}\rangle_{(c)}=\frac{1}{\hat{\rho}} \sum_{i \in \mathcal{S}_{c}} w^{(i)} \hat{\phi}^{(i)},
$$

where $\hat{\phi}^{(i)}$ indicates $\phi$ evaluated by $i$ th particle, $\mathcal{S}_{c}$ is set of particles in the cell $(c)$ and

$$
\hat{\rho}=\frac{1}{\delta V_{c}} \sum_{i \in \mathcal{S}_{c}} w^{(i)}
$$

is the estimated density based on the cell volume $\delta V_{c}$. 
In comparison to conventional Langevin simulations, here the macroscopic coefficients $\{c, \gamma, \hat{c}, \hat{\gamma}\}$ which close the drift term are computed from linear systems (3.27) for each computational cell and per each time step. Hence correct relaxation rates in the velocity update besides accurate collisional transfer in the position update are obtained.

\subsection{Discretization of the screened-Poisson model}

In order to translate the long-range interactions into a suitable elliptic problem, first we need to represent Eq. (3.30) in a bounded domain $\Omega_{x} \subset \mathbb{R}^{3}$. Following uniqueness of the solution, the SP equation can be reset in a bounded domain $\Omega_{x}$ as

$$
\left\{\begin{array}{ccc}
\left(\Delta-\lambda^{2}\right) \tilde{\Phi}_{r>0} & =-n & \text { in } \Omega_{x} \\
\tilde{\Phi}_{r>0} & =\tilde{\Phi}_{D} & \text { on } \partial \Omega_{x},
\end{array}\right.
$$

where the Dirichlet condition $\tilde{\Phi}_{D}$ is evaluated via

$$
\tilde{\Phi}_{D}(x)=\int_{r>0} G(r) n(y) d y, \quad \forall x \in \partial \Omega_{x} .
$$

The boundary term is computed numerically using quadrature estimation of the Vlasov integral and introducing a cut-off to contain the computational cost, i.e., $r \in\left(0, r_{\text {cut }}\right)$. Note that the singularity in $G(r)$ is avoided once the integral is formulated in the spherical coordinates. Here, we have set $r_{\text {cut }}=3 \sigma$ and used $N_{\text {quad }}$ Legendre-Gauss quadrature points for the numerical integration. Once the values of the unique global solution on the boundary $\partial \Omega_{x}$ is computed, this elliptic differential equation on the bounded domains can be solved for the interior of the domain using standard numerical methods. In $\S \mathrm{B}$, we present a short description for the adopted Hybridized Discontinuous Galerkin solver. Once our estimate of the attractive force at time step $(n)$ is obtained, the velocity update takes the form

$$
V^{(n+1)}=V^{(*)}+\hat{H}^{(n)}\left(X^{(n)}\right) \Delta t,
$$

where $\hat{H}^{(n)}\left(X^{(n)}\right)$ is the approximated attractive force at the particle position $X^{(n)}$ (see Appendix $\S \mathrm{B}$ for details).

\subsection{Solution algorithm}

It is evident from time-stepping scheme given by Eqs. (4.2a)-(4.2b) that the evolution of position and velocity of particles is decoupled during each time step. While more accurate schemes such as those discussed by Jenny et al. (2010); Gorji et al. (2011); Jenny \& Gorji (2019) can be devised for a joint position-velocity update, here for simplicity the decoupled version is utilized. Note that as shown by Pfeiffer \& Gorji (2017); Jun et al. (2019), this decoupling is accurate and justified as long as the gradients of macroscopic fields are resolved. In comparison to DSMC (Bird 1994), where the collisional scales have to be resolved, here we obtain a significant computational advantage by relaxing the discretization constraints.

Algorithm 1 provides a summary of the DFP-SP solution method for simulations of multi-phase flows. Prior to the simulation, the domain is discretized into a set of computational cells. Furthermore an appropriate time step size and particle weight are chosen. After initializing the particles according to their weights and initial density field, the particles evolve according to Alg.1. Note that particles boundary conditions are implemented similar to DSMC, where depending on the distribution of incoming or reflected particles, open or wall boundaries are handled, respectively. 


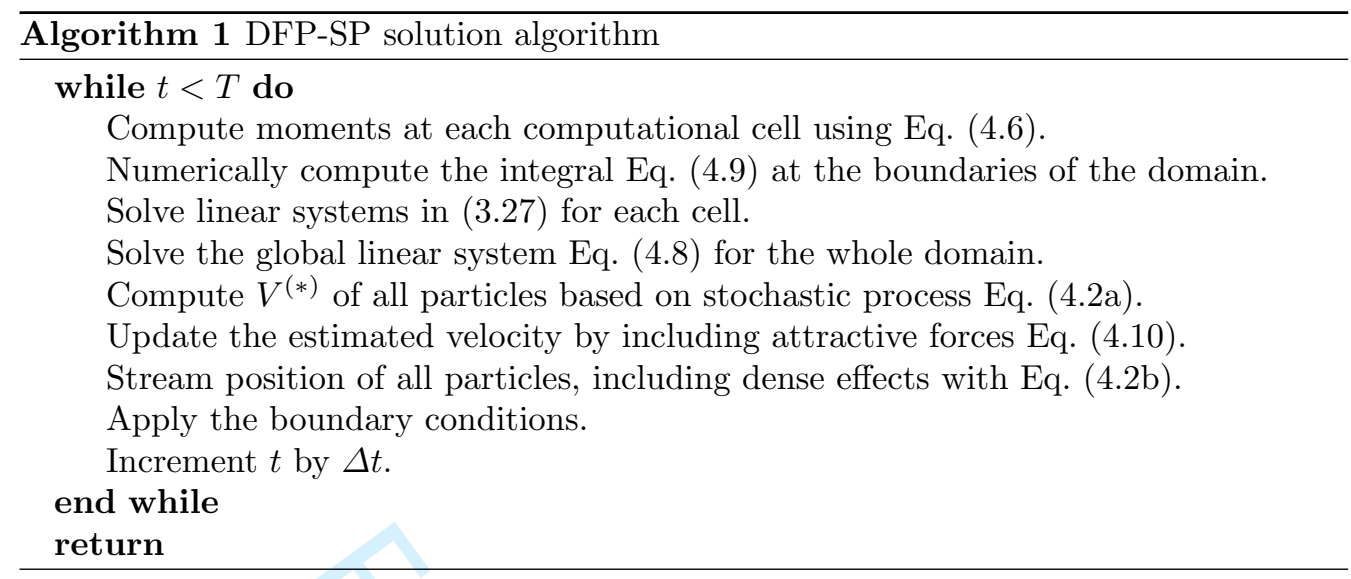

\section{Validation results}

For validation of the devised DFP-SP solution algorithm, we focus on evaporation of liquid Argon slab once in contact with the equilibrium vapor and once in contact with the vacuum. The coefficients of the Sutherland potential $\phi(r)$ are chosen according to $\sigma=3.405 \times 10^{-10} \mathrm{~m}$ and $\phi_{0}=4.17 \epsilon$ where $\epsilon=119.8 k_{b} \mathrm{~kg} \cdot \mathrm{m}^{2} \cdot \mathrm{s}^{-2}$ (provide citation) . Fitting $\tilde{\phi}(r)$ to the attractive part of the Sutherland potential leads to the coefficients $a=-1.64835851 \times 10^{-28} \mathrm{~kg} \cdot \mathrm{m}^{2} \cdot \mathrm{s}^{-2}$ and $\lambda=6.91304716 \times 10^{9} \mathrm{~m}^{-1}$.

We examine accuracy of the DFP-SP model in predicting capillary properties of droplets, such as the surface tension and the evaporation rate. The results are then compared with benchmark Monte-Carlo simulations of the Enskog-Vlasov equation (ESMC-Vlasov). For implementation details of the benchmark ESMC-Vlasov approach see Appendix $\S$ A.

The time step size satisfying a Courant-Friedrichs-Lewy (CFL) type condition

$$
\begin{aligned}
\Delta t & \leqslant \frac{\min \left(\lambda^{(0)}, h\right)}{\max \left(\sqrt{k T^{(0)} / m}\right)}, \\
\text { where } \quad \lambda^{(0)} & =\frac{1}{\sqrt{2} \pi \sigma^{2} n^{(0)} Y\left(n^{(0)}\right)}
\end{aligned}
$$

is chosen. Here, $\lambda$ provides an estimate of the mean free path, $h$ indicates the mesh size, and superscript $(.)^{(0)}$ denotes the local initial value. For further details see Pfeiffer \& Gorji (2017).

\subsection{Evaporation at equilibrium}

In order to present DFP-SP prediction of the surface tension, evaporation of a liquid Argon slab in contact with the vapor phase at equilibrium is studied. In particular, we consider the phase transition of the liquid slab located within $[0,1] \times[0,30 \sigma] \times[0,1] \mathrm{m}^{3}$ which is surrounded by the vapour confined in $[0,1] \times[0, L] \times[0,1] \mathrm{m}^{3}$ with $L=90 \sigma$. Since only $x_{2}$ direction is relevant, the other dimensions in the physical space are ignored in the evolution of particles positions. The liquid slab and the vapour around it, are initialized with number densities $n_{\text {liq }}$ and $n_{\text {vap }}$, respectively. In order to maintain the stationary scenario, the liquid slab is heated up every $N_{\text {therm }}$ steps, where the particles inside $x_{2} \in\left[L / 2-L_{\text {therm }} / 2, L / 2+L_{\text {therm }} / 2\right]$ are thermostated with the initial temperature 
$T^{(0)}$. This is achieved by re-scaling particles fluctuating velocities. Furthermore, the specular reflection boundary conditions are deployed here.

After conducting convergence studies, it is found that $N_{\text {cell }}=180$ computational cells and the CFL number 0.01 provide converged results. Moreover, a fixed statistical weight of $w=10^{14}$ was picked for the test cases with $T^{(0)} \in\{150,160,170,180\} \mathrm{K}$, $n_{\text {liq }}^{(0)} \in\{0.6,0.526,0.45,0.36\} \times \sigma^{-3} \mathrm{~m}^{-3}$ and $n_{\text {vap }}^{(0)} \in\{0.05,0.077,0.12,0.15\} \times \sigma^{-3} \mathrm{~m}^{-3}$. The stationary distribution is obtained after $5^{\prime} 000$ steps and the time averaging is performed over $15^{\prime} 000$ steps onward. Here, $L_{\text {therm }}=10 \sigma$ was chosen as the length of thermostated sub-domain which was carried out once every $N_{\text {therm }}=1^{\prime} 000$ steps. The initial densities of vapour and liquids for each simulation were picked such that unstable regions of the phase diagram are not met, as depicted in Fig. 3.

For the liquid slab at equilibrium, the surface tension coefficient $\gamma$ is defined as a norm of the difference between diagonal element of the kinetic pressure tensor and the equilibrium kinetic pressure across the phase transition

$$
\gamma=\frac{1}{2} \int_{0}^{L}\left(p_{22}-p_{0}\right) d x_{2} .
$$

For details see Ghoufi \& Malfreyt (2010); Hirschfelder et al. (1964). The integration over the domain can be taken numerically, since the discrete values of the pressure tensor are estimated at every cell.

Comparison between simulation results obtained from the DFP-SP model with respect to the benchmark ESMC-Vlasov is shown in Fig. 1. Although the DFP-SP model under-predicts the peak in estimating $\left|p_{22}-p_{0}\right|$ at the interface, overall a very good agreement between the two is observed. Note that the discrepancy in $\gamma$ between both methods increases as the liquid gets denser at lower temperatures. Furthermore, the computational costs of the discussed simulation settings are presented in Fig 2. The cost independence of the DFP-SP solution algorithm from the density is clearly visible.

\subsection{Evaporation to vacuum}

Since streaming of the stochastic particle in the DFP model is consistent with the Enskog equation only on the moment level, the measurement of evaporation rate based on particle trajectory as suggested in Yasuoka et al. (1994); Kon et al. (2014); Kobayashi et al. (2016) would not lead to an accurate estimation of the desired property. Alternatively, the evaporation rates can be approximated via moments as a liquid slab evaporates into the vacuum, see Frezzotti et al. (2005). The outflow mass flux $\dot{m}_{\text {out }}$ is indeed a function of the evaporation rate $\sigma_{e}$

$$
\dot{m}_{\text {out }}=A \sigma_{e} n_{g}\left(T_{L}\right) \sqrt{\frac{k_{b} T_{L}}{2 \pi m}},
$$

where $A$ indicates the area of cross-section normal to the inter-phase, $n_{g}\left(T_{L}\right)$ is the number density of the gas computed at the temperature of the liquid phase $T_{L}$. Hence, validation for the evaporation rate translates into the consistency of temperature and density profiles, as well as the outgoing mass flux with respect to the benchmark solution. Except for the boundary conditions, we consider similar simulation settings as the test case with the evaporation of liquid at equilibrium, i.e., $\S 5.1$. Particles are re-initialized in the liquid slab as they leave the domain with the new position sampled randomly 


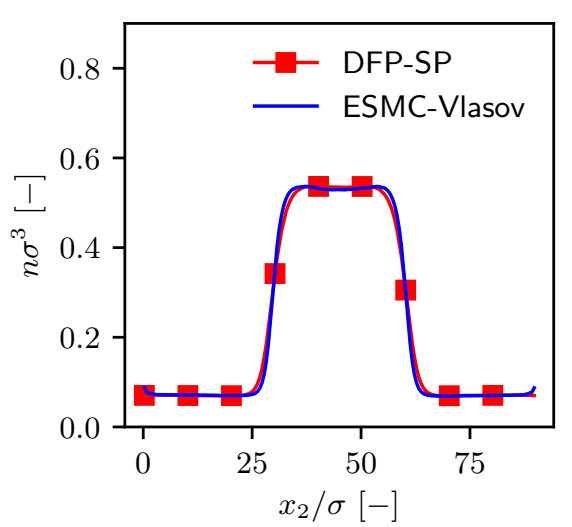

(a)

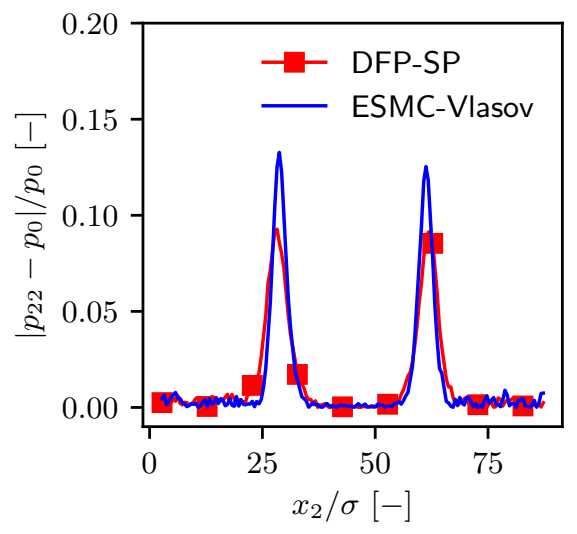

(c)

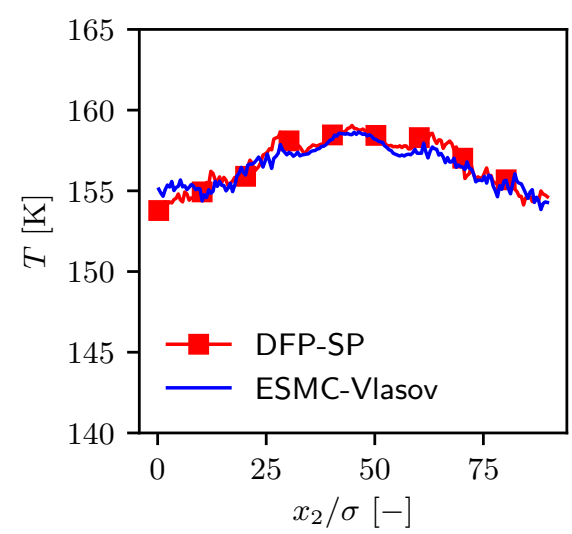

(b)

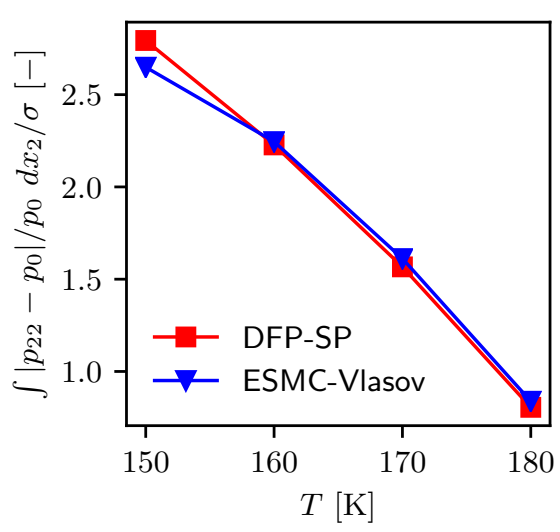

(d)

Figure 1: Phase transition of Argon at $T^{(0)}=160 \mathrm{~K}$. (a) Normalized number density. (b) Normalized temperature. (c) Normalized difference between diagonal and equilibrium kinetic pressure. (d) Normalized surface tension for liquid temperatures of $T^{(0)} \in$ $\{150,160,170,180\} \mathrm{K}$.

from a uniform distribution on the interval $x_{2} \in\left[L / 2-L_{\text {therm }} / 2, L / 2+L_{\text {therm }} / 2\right]$. For the re-initialized particle, the velocity component in the relevant flow direction, i.e., $V_{2}$, is sampled again from an equilibrium distribution with mean and temperature of the local computational cell, as the other components of the velocity remain unchanged.

In comparison to ESMC-Vlasov, we obtain accurate predictions of temperature, density, velocity and the outflow mass flux, as shown in Fig. 4. Similar test cases were performed at other temperatures and again good agreements between the DFP-SP model and the benchmark were observed, indicating consistent evaporation rates.

\section{Inverted temperature gradient}

One of the interesting examples where counter-intuitive non-equilibrium effects become evident is the inverted temperature profile in the vapor phase contained between two liquid slabs at different temperatures. First, Pao (1971) found out that the temperature 


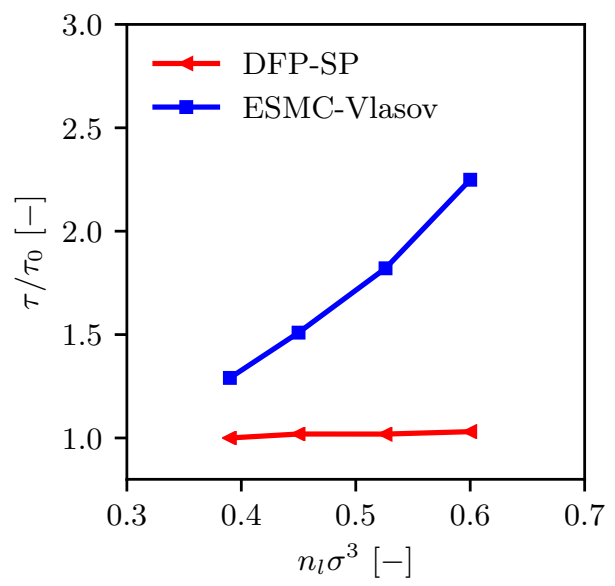

Figure 2: Execution time per particle $\tau:=t^{\text {ex. }} / N_{p}$ for evaporation simulation at the equilibrium using ESMC-Vlasov and DFP-SP solution algorithms. The cost is normalized by $\tau_{0}$ denoting DFP-SP execution time for the case $T^{(0)}=180 \mathrm{~K}$.

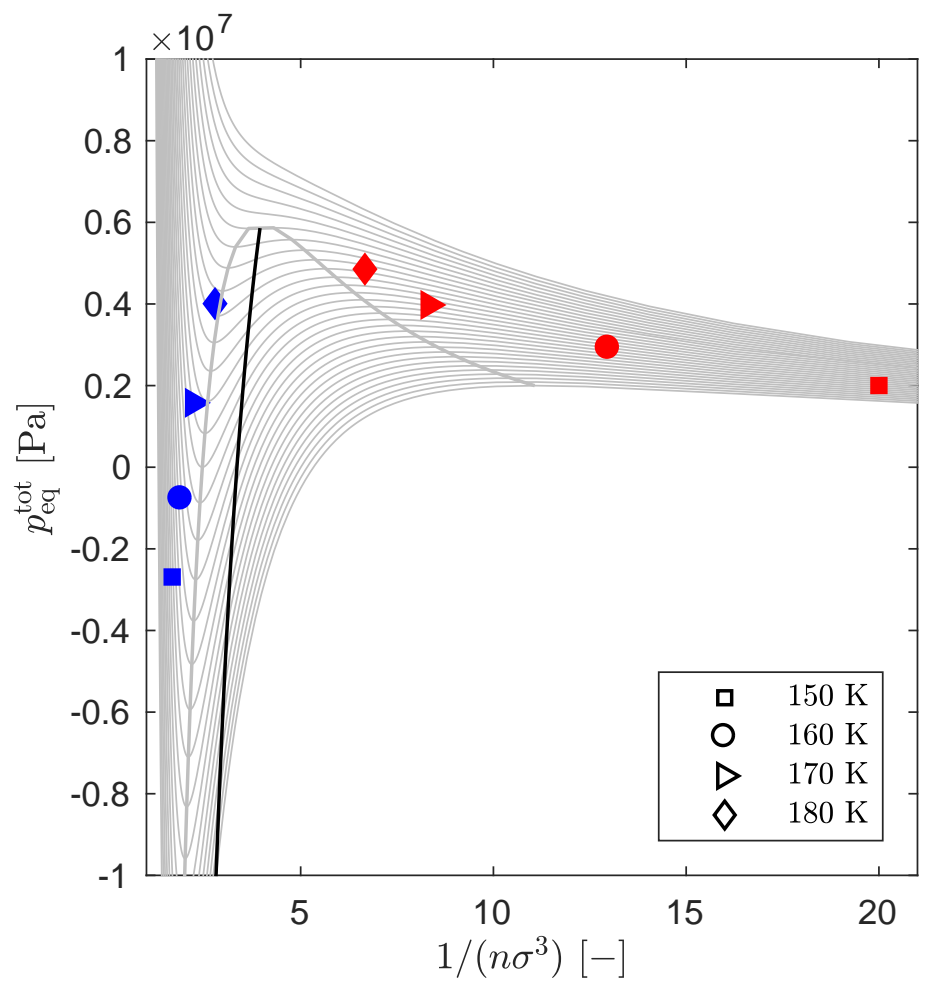

Figure 3: Contours of total equilibrium pressure $p_{\mathrm{eq}}^{\text {tot }}(n, T)$ at constant temperatures as well as the initial state pairs $\left(n_{\text {liq }}, T_{\text {liq }}\right)$ and $\left(n_{\text {vap }}, T_{\text {vap }}\right)$ for the evaporation test case with initial temperatures of $T^{(0)} \in\{150,160,170,180\} \mathrm{K}$ and corresponding vapour (in red) and liquid (in blue) number densities of $n_{\text {liq }}^{(0)}=\{0.6,0.526,0.45,0.36\} \times \sigma^{-3} \mathrm{~m}^{-3}$ and $n_{\text {vap }}^{(0)}=\{0.05,0.077,0.12,0.15\} \times \sigma^{-3} \mathrm{~m}^{-3}$, respectively. 


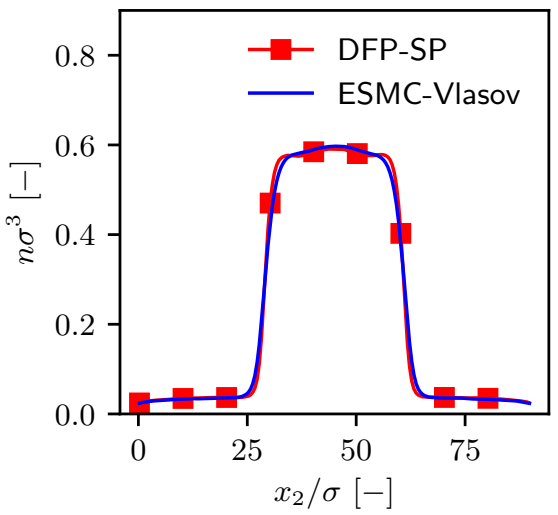

(a)

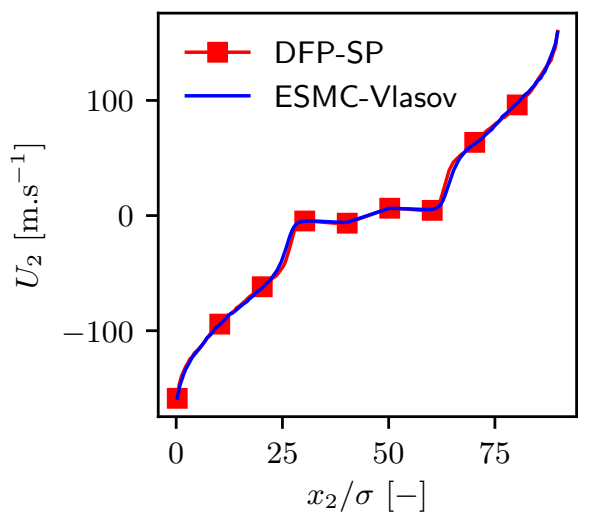

(c)

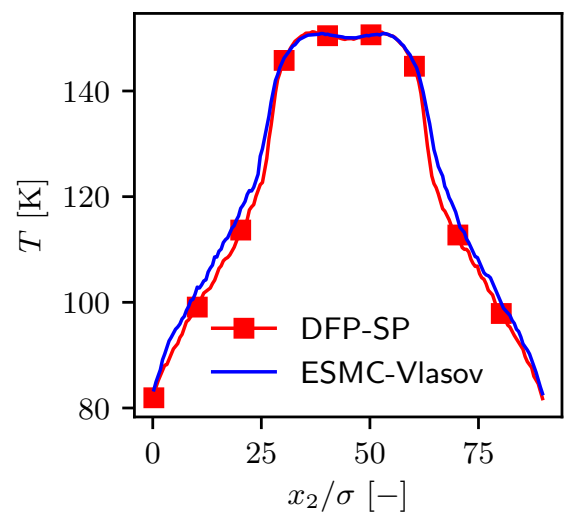

(b)

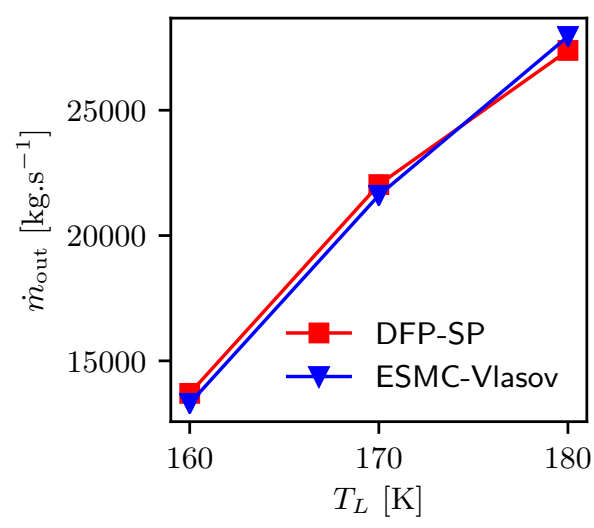

(d)

Figure 4: Simulation results for evaporation of liquid Argon into the vacuum obtained from ESMC-Vlasov and DFP-SP models. (a) Normalized number density. (b) Temperature. (c) Mean velocity. (d) Outflow mass flux.

profile does not increase monotonically from cold to hot liquid, suggesting an inverted temperature profile in the vapor phase. Since then, this phenomenon has been studied and validated in details using tools across Molecular Dynamics and kinetic theory (see e.g. Frezzotti et al. (2003); Meland (2003); Meland \& Ytrehus (2004)).

We tackle this problem by the devised DFP-SP model, and validate our results with respect to the ESMC-Vlasov approach. Consider a solution domain in the physical space $\Omega=[0,1] \times[0, L] \times[0,1] \mathrm{m}^{3}$ where $L=70 \sigma$. Cold and hot liquid slabs are located at $x_{2} \in\left[0, L_{c}\right]$ and $x_{2} \in\left[L-L_{h}, L\right]$, respectively, with $L_{c}=L_{h}=15 \sigma$. The thermodynamic states of cold and hot slabs are initially set by the density-temperature pairs $\left(n_{c}, T_{c}\right)$ and $\left(n_{h}, T_{h}\right)$, respectively. The vapour with initial density-temperature of $\left(n_{v}, T_{v}\right)$ fills the space between two slabs as depicted schematically in Fig. 5. In order to keep temperature of the liquid slabs close to the initial setting, particles within $x_{2} \in\left[0, L_{\text {therm }}\right]$ and $x_{2} \in\left[L-L_{\text {therm }}, L\right]$, where $L_{\text {therm }}=15 \sigma$, are thermostated to the initial temperature every $N_{\text {therm }}$ steps. This is done by re-scaling particles fluctuating 


$\begin{array}{cccccc}\text { Test Case } & n_{c} \sigma^{3}[-] & n_{h} \sigma^{3}[-] & n_{v} \sigma^{3}[-] & T_{c}[\mathrm{~K}] & T_{h}[\mathrm{~K}] \\ \text { (I) } & 0.42 & 0.40 & 0.13 & 170 & 172 \\ \text { (II) } & 0.45 & 0.43 & 0.12 & 167 & 169 \\ \text { (III) } & 0.47 & 0.45 & 0.10 & 165 & 167\end{array}$

Table 1: Initial number density and temperature of liquid slabs and the vapour for the inverted temperature gradient simulations

velocities. Due to symmetry of the simulation setting, position evolution of the particles is considered only along the $x_{2}$-coordinate.

Fully diffusive scattering is applied as the boundary condition to the both ends of the domain. As particles leave the domain, their velocities are sampled from the flux of the Maxwellian distribution with the initial temperature of the nearest liquid slab. Next the scattered particles are streamed into the domain for the remaining fraction of the time step.

Since there exists a mass flux from hot slab towards to the cold one, the system does not admit a stationary state. Hence time averaging can not be deployed to improve the estimate of observables. However, by moving the observer in the direction opposite to the mass flux, a quasi-stationary condition is reached where time averaging becomes applicable. Notice that a similar approach is typically utilized for one-dimensional shock wave problems (Bird 1994; Gorji \& Jenny 2013). Once every $N_{\text {shift }}$ steps, the simulation frame is moved by $\Delta / 2$ where $\Delta:=\left(\mathcal{V}_{c}-\mathcal{V}_{h}\right), \mathcal{V}_{c}:=x_{L}$ and $\mathcal{V}_{h}:=\left(L-x_{R}\right)$. Here $x_{R}$ is the right-most location with number density $0.9 n_{h}$, whereas $x_{L}$ is the left-most position with number density $0.9 n_{c}$. As the simulation frame is moved, the particles which appear outside of the domain are removed. Moreover, new particles are generated to fill the void space. Velocities of the newly introduced ones are sampled from the Maxwellian distribution with temperature of the nearest liquid slab. Their corresponding positions are sampled uniformly inside the void space.

Three settings with temperatures below the critical point are considered as presented in Table 1. The temperature difference between liquid slabs is set to $2 \mathrm{~K}$. After a convergence study, a uniform discretization of physical space with mesh size of $\Delta x_{2}=\sigma / 2$ along $x_{2}$ direction as well as the CFL number 0.01 are picked due to their reasonable accuracy. In all simulations, initially 10,000 particles per cell for the vapour phase are generated, leading to the statistical weights $w \in\{4.3,5.1,5.6\} \times 10^{13}$ associated with cold liquid slab temperatures $T_{c} \in\{165,167,170\} \mathrm{K}$, respectively. Furthermore, the domain is shifted every $N_{\text {shift }}=500$ steps and the liquid slabs are thermostated every $N_{\text {therm }}=1^{\prime} 000$ steps, for all considered cases.

As depicted in Fig. 6, the DFP-SP solution algorithm approximates the density, the mean velocity, the temperature and the heat-flux of the considered test cases accurately in comparison to the benchmark. The inverted temperature gradients are well recovered by the presented DFP-SP model. Furthermore, the profiles near the phase-transition regimes are found to be well approximated. As suggested by Meland \& Ytrehus (2004), since the colder liquid slab has smaller evaporation and condensation rates, we observe 


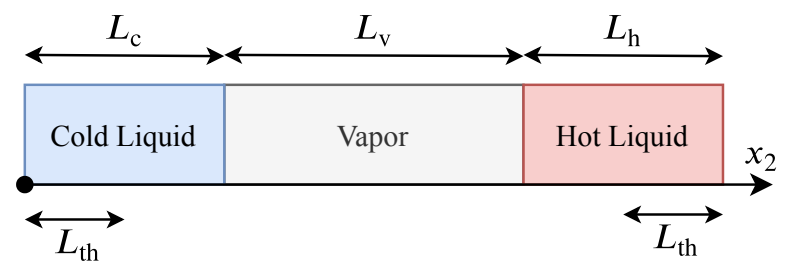

Figure 5: Schematic of the inverted temperature gradient simulation

an accumulation of hot particles near the colder liquid slab. This leads to the conclusion that particles emitted from the hot liquid slab plays a more important role behind this counter intuitive non-equilibrium temperature profile.

In order to obtain a better understanding of the underlying phase-transition dynamics, let us consider the case where a single liquid slab distributed in $x_{2} \in[0,10 \sigma]$ is in contact with a diffusive wall at $x_{2}=0$ and is surrounded by the vapour distributed inside $x_{2} \in[10 \sigma, 20 \sigma]$. For simplicity, we consider the specular wall at the end of domain $x_{2}=20 \sigma$ to contain the vapor. We simulated this setting using the DFP-SP model for liquid temperatures considered in the inverted-temperature-gradient test case using $\omega=4 \times 10^{13}$, CFL number 0.01 and $\Delta x=\sigma / 2$. As shown in Fig. 7 , the temperature of the fluid first drops during the transition of the liquid to the vapor and then raises back inside the vapor phase. It is important to notice that the further we move far from the droplet, the more increase we observe in the temperature. This can be explained by noting that at relatively low vapor pressure, high energy particles can travel longer distances before reaching equilibrium with the background gas. Here, one can make three observations. First, note that as the temperature of the liquid decreases, the difference between temperature of the liquid and its surrounding vapour becomes larger. Next, the temperature drop gets more pronounced, once colder liquid slabs are considered. Finally, by a simple super-position of the observed single liquid slab temperature profiles, we get a qualitative picture of the inverted temperature gradient behaviour consistent with our two liquid slabs system shown in Fig. 6.

\section{Spinodal decomposition}

Phase transition phenomena which are pronounced in breakup and coalescence of bubbles as well as droplets, comprise one of grand challenges in simulation of multi-phase flows. In particular, an accurate description of these processes demands incorporation of mass, momentum and energy transfer among different phases, if conventional multi-phase solvers are considered (Shan \& Chen 1993; Watanabe et al. 2012). However due to the fact that the considered EV kinetic framework provides a universal flow description across liquid and gas phases, a systematic exchange of mass, momentum, and energy at the interface is obtained naturally, consistent with the underlying molecular potential. In order to demonstrate the potential of the devised kinetic model in simulating such scenarios, here we study spinodal decomposition of Argon, where formation of droplets as well as bubbles can be investigated.

Let us consider a simulation domain $\Omega_{x}=[0, L]^{2} \times[0,1] \mathrm{m}^{3}$ where $L=5 \times 10^{-8} \mathrm{~m}$. Furthermore, assuming symmetry in the third dimension, we intend to study a twodimensional (2D) planar fluid flow in $\Omega_{x}$ that, after a convergence study, is discretized with $250 \times 250 \times 1$ cells. As boundary conditions for relevant dimensions, i.e. $x_{1}$ and 

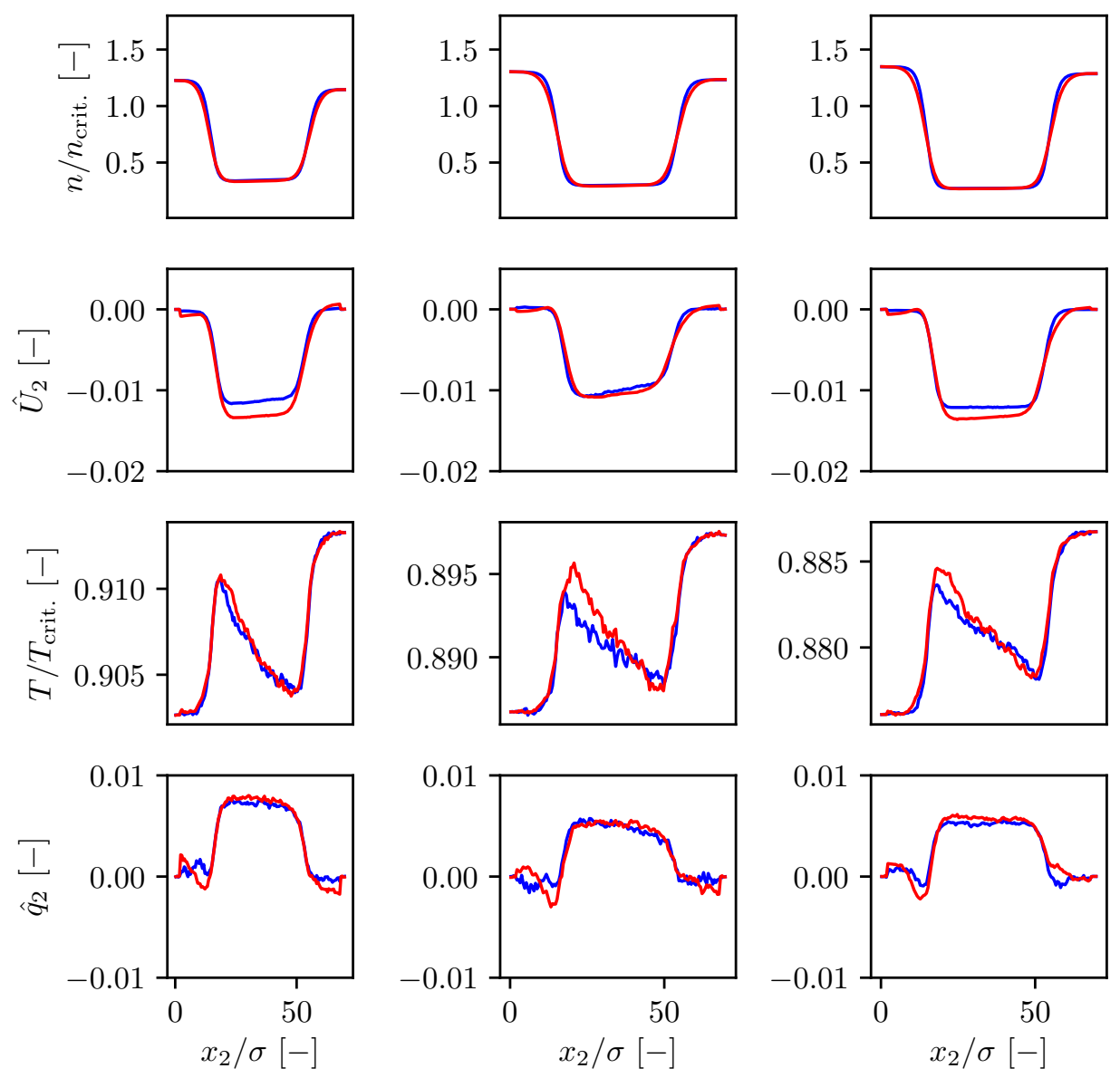
(a) Test case (I)
(b) Test case (II)
(c) Test case (III)

Figure 6: Normalized number density, mean velocity, temperature, and heat-flux obtained from inverted temperature gradient simulations using ESMC-Vlasov (blue) and DFP-SP (red) solution algorithms. The critical point $\left(n_{\text {crit. }}, T_{\text {crit. }}\right)=\left(0.3568 / \sigma^{3} \mathrm{~m}^{-3}, 188.33 \mathrm{~K}\right)$ is used to normalize density and temperature. Heat-flux and mean velocity are normalized by $\hat{q}_{2}:=q_{2} /\left(\rho_{c}\left(k T_{c} / m\right)^{3 / 2}\right)$ and $\hat{U}_{2}:=U_{2} / \sqrt{k T_{c} / m}$, respectively.

$x_{2}$, we consider specular reflection for particles that leave the domain. This leads to the Neumann boundary condition for the SP model owing to long-range interaction, i.e., $\tilde{n}_{i} \partial \tilde{\Phi} / \partial x_{i}=0$, where $\tilde{n}$ indicates the unit outward normal vector on the surface of the boundary $\partial \Omega_{x}$.

First, particles are initialized inside the domain $\Omega_{x}$ with a uniform distribution in physical space and Maxwellian in velocity for a given initial number density $n^{(0)}$ and temperature $T^{(0)}$. The idea is to pick an unstable state $\left(n^{(0)}, T^{(0)}\right)$ from a point on the phase diagram that does not belong to liquid nor vapour at the equilibrium. Then, we let the system relaxes towards the equilibrium. The expectation is that particles move away from this unstable initial condition until the corresponding mixture reaches a stable state at the equilibrium on the binodal diagram. Here we considered three test cases, 


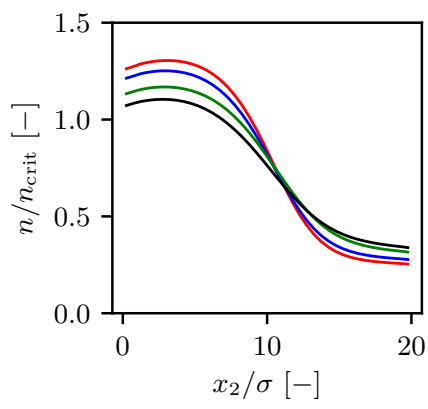

(a) Number density

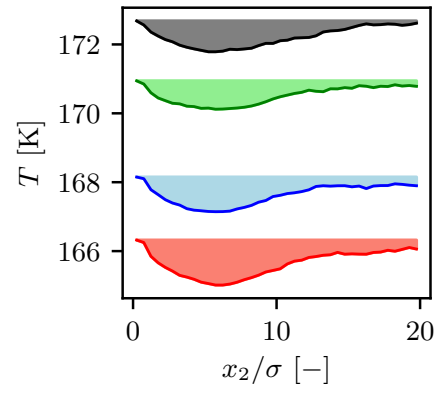

(b) Temperature

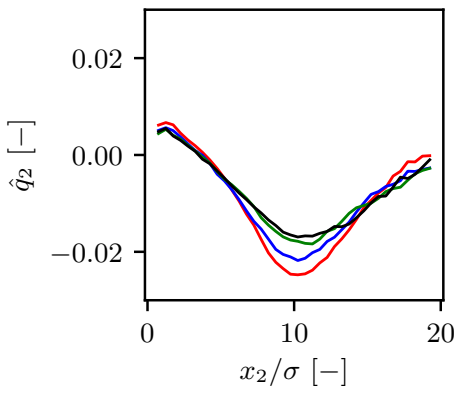

(c) Heat-flux

Figure 7: Single droplet evaporation. The profile of number density, temperature and heat-flux for the liquid/vapour contained between diffusive and specular walls.

Test Case

(A) Low density

(B) Medium density

(C) High density

$$
n^{(0)}\left[\mathrm{m}^{-3}\right] \quad T^{(0)}[\mathrm{K}]
$$

$3 \times 10^{27}$

$5 \times 10^{27}$

$8 \times 10^{27}$
120

120

120
$N_{p}$

$\begin{array}{cc}10^{-14} & 1.33 \times 10^{8} \\ 10^{-14} & 1.25 \times 10^{8} \\ 2 \times 10^{-14} & 1.33 \times 10^{8}\end{array}$

Table 2: Simulation setting of the spinodal decomposition. Initial number density $n^{(0)}$, temperature $T^{(0)}$, the time step size $\Delta t$ and number of particles $N_{p}$ used in the simulations are reported here.

as detailed in Table 2, with the main difference in their initial densities. The intention behind these cases is to study formation of droplets in (A) low density range and (B) medium density range. Furthermore formation of bubbles is tackled in a high density range test case of $(\mathrm{C})$.

\section{Test case (A): formation of nano droplets}

As shown in Movie 1 and depicted in Fig. 8, formation, and coalescence of droplets can be observed following the evolution of the number density. Note that two droplets, designated by rings around them and identified in the figure, are tracked in time for further analysis.

As shown in Fig. 9, unless interrupted by a merge with another droplet, the radius of both droplets first increases with the rate of $t^{1 / 3}$ and then their growth rate reduces to $t$. At the first stage, when the inertia of the fluid system is negligible, the Lifshitz-Slyozov mechanism is dominant (Siggia 1979). This mechanism gives the growth rate of $t^{1 / 3}$ consistent with our result. Next, once we reach critical quenches (similar volume fraction of both phases), the growth rate stabilizes at the growth rate of $t$ (Siggia 1979; Bastea \& Lebowitz 1997).

Furthermore, statistics of number density, temperature, and velocity magnitude is Cambridge University Press 
shown in Figs. 10 and 11a. Moreover, we study the total energy of the system

$$
E_{\text {total }}(t)=\underbrace{\frac{1}{2} \int_{\Omega_{x}} \rho(x, t) \mathbb{E}\left[V_{j} V_{j} \mid x, t\right] d x}_{E_{\text {kin }}(t)}+\underbrace{\frac{1}{2 m} \int_{\Omega_{x}} \rho(x, t) \tilde{\Phi}(x, t) d x}_{E_{\text {pot }}(t)}
$$

as derived in Appendix $\S \mathrm{C}$, and here is evaluated numerically from the simulation results. As shown in Fig. 11b, we observe energy transformation from potential to kinetic at the early stage of the spinodal decomposition, while the total energy stays almost constant. Furthermore, we observe relatively small decay of the total energy of the system, which is due to numerical errors in the deployed time integration scheme.

\section{Test case (B): formation of larger droplets}

Since here the initial unstable state has a larger density with respect to the case (A), more sizable droplets are formed as illustrated in the evolution of the number density in Movie 2 and Fig. 12. The maximum, median, and minimum values of number density, temperature, velocity magnitude as well as kinetic, potential, and total energy are computed. The results are shown in Figs. 13 and 14b. Unlike the test case (A), we observe the appearance of more nano droplets in the early stage of the spinodal decomposition, which leads to the subsequent formation of larger droplets as coalescence among more nano droplets occurs.

\section{Test case (C): formation of nano bubbles}

Having initialized spinodal decomposition with a large enough density, here we observe the formation of bubbles instead of droplets, as illustrated in the evolution of the number density in Movie 3 and Fig. 15. Similar to the last two test cases, statistical investigation of number density, temperature, velocity magnitude as well as kinetic, potential, and total energy are shown in Figs. 16 and 17b.

\section{Computational cost:}

As expected, the computational cost of DFP-SP simulations remains identical, as the deployed spatial discretization as well as particle numbers among considered spinodal decomposition test cases are similar. However, once the liquid is formed, the average execution time per 100 iterations ranges from $360 \mathrm{~s}$ to $540 \mathrm{~s}$, which can be explained by the in-homogeneity of the load balance as particles cluster inside the liquid phase. Note that here the parallelization is based on a domain decomposition of the physical space. For the simulations of the spinodal decomposition, we deployed 40 cores of Intel(R) Xeon(R) Platinum $8160 \mathrm{CPU} @ 2.10 \mathrm{GHz}$ in parallel. Using this computational resource, the total computation time, e.g. for case $(\mathrm{C})$, with end time of $t_{\text {final }} \approx 1.3$ ns was about 4 days.

To further demonstrate the capability of the devised DFP-SP model to address realistic and challenging multi-phase flows, here we extend the study of the spinodal decomposition to the three dimensional (3D) physical space. The simulation domain is set to $\Omega_{x}=[0, L]^{3} \mathrm{~m}^{3}$ where $L=3 \times 10^{-8} \mathrm{~m}$. Here, $\Omega_{x}$ is discretized by $100^{3}$ cells. As boundary conditions, specular reflection for particles leaving the domain as well as the Neumann boundary condition for the SP model of long-range interaction, is considered. Here, $N_{p}=4.32 \times 10^{8}$ particles and time step size of $\Delta t=2 \times 10^{-14}$ s are considered. Initially, we sample the position of particles inside $\Omega_{x}$ from a uniform distribution with a weight that provides us with the initial number density of $n^{(0)}=8 \times 10^{27} \mathrm{~m}^{-3}$. 


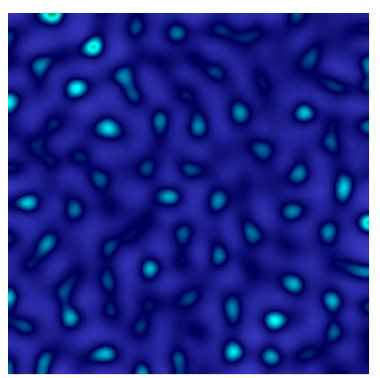

$t=0.15 \mathrm{~ns}$

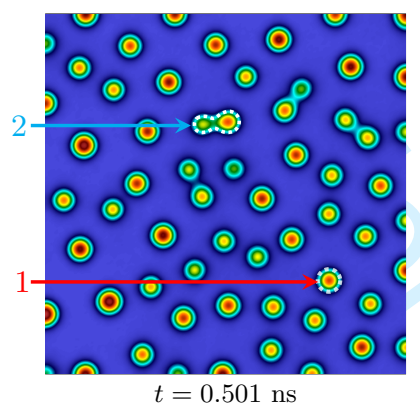

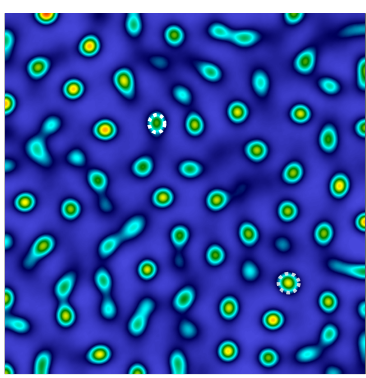

$t=0.201 \mathrm{~ns}$

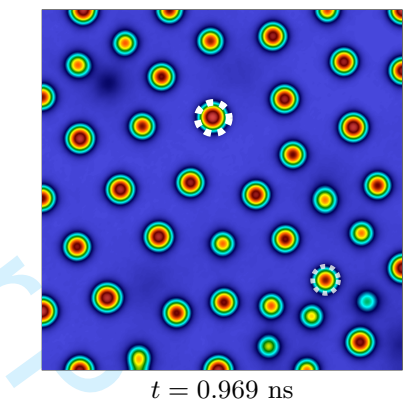

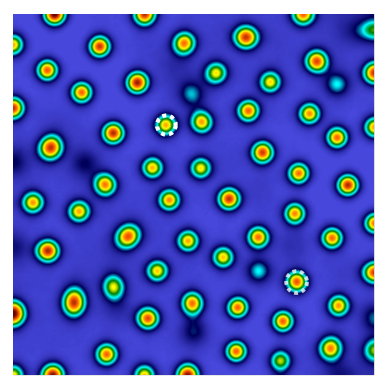

$t=0.312 \mathrm{~ns}$

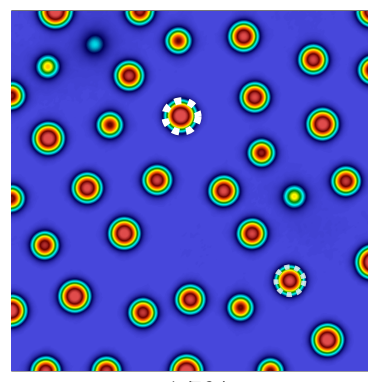

$t=1.764 \mathrm{~ns}$

Figure 8: Evolution of normalized number density in the spinodal decomposition of Argon obtained from simulation results of the DFP-SP model for test case (A), with initial number density $n^{(0)}=3 \times 10^{27} \mathrm{~m}^{-3}$ and temperature $T^{(0)}=120 \mathrm{~K}$ in the domain $\Omega_{x}=[0, L]^{2} \times[0,1] \mathrm{m}^{3}$ where $L=5 \times 10^{-8} \mathrm{~m}$. Here, two droplets are labeled with rings around them which are tracked in time for further analysis.

Furthermore, similar to the 2D case, the velocity of particles are initialized by sampling from the Maxwellian distribution such that we obtain the initial temperature of $T^{(0)}=120 \mathrm{~K}$.

Having employed similar computational resources like the one for $2 \mathrm{D}$ case, we observe that the average execution time for 50 iterations per cell approximately ranges from $600 \mathrm{~s}$ to $2040 \mathrm{~s}$. Here, the effect of load imbalance due to particle clustering is more pronounced. Hence, we acknowledge the need for devising an efficient load balancing scheme that benefits from density distribution in the physical space, in parallel computations of multi-phase flows using particles. Similar to the test case $(\mathrm{C})$, as shown by the evolution of number density in Fig. 18 and more profoundly in Movie 4, we observe the formation of bubbles contained in the liquid. Simulation result of this spinodal decomposition using DFP-SP model was carried out until $t_{\text {final }} \approx 0.304$ ns with time step size of $\Delta t=0.02 \mathrm{ps}$ which took about 3 days.

\section{Conclusion and outlook}

Multi-phase flows far from the equilibrium demand for high-fidelity models beyond the conventional hydrodynamics. This study lays out a Fokker-Planck-Poisson approach which systematically reduces the multi-phase kinetic description into a set of SDEs suitable for efficient particle simulations. The short-range molecular collisions are described by the dense FP model, whereas the long-range interactions are cast into a SP elliptic model. The resulting system then is simulated using a particle Monte-Carlo scheme in 


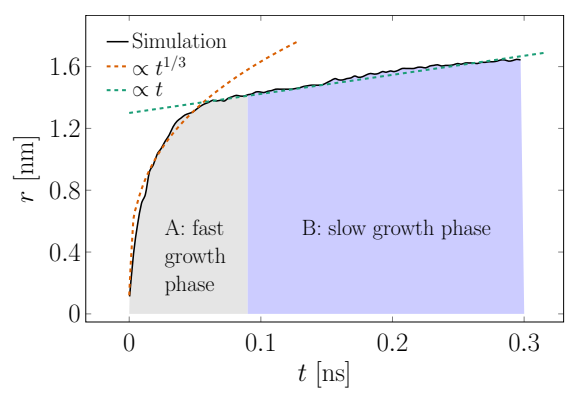

(a) Droplet \#1

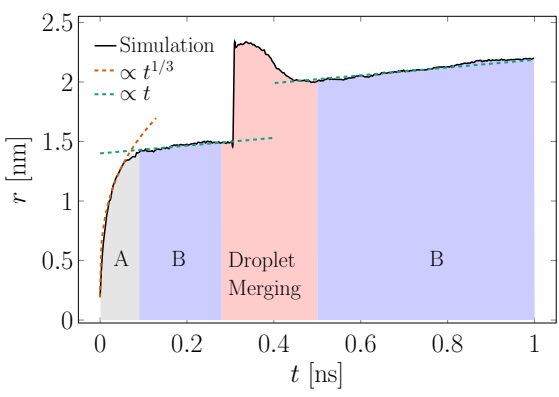

(b) Droplet \#2

Figure 9: Growth of the labeled droplets, i.e., droplet \#1 in (a) and \#2 in (b) as identified in Fig. 8, are studied by investigating the evolution of droplet's radius $r$ in time. We observe that initial growth of radius in both cases is $\propto t^{1 / 3}$ which follows by $\propto t$ unless interrupted by a coalescence, e.g. in (b).

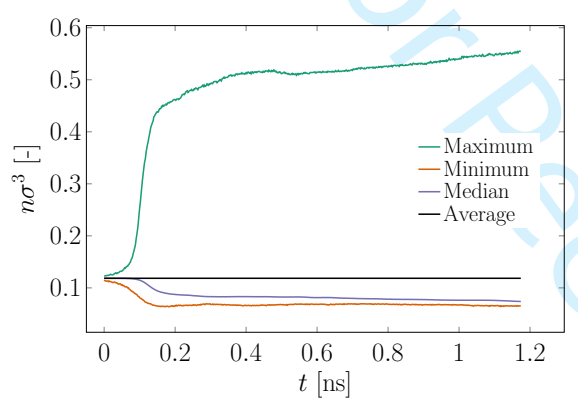

(a)

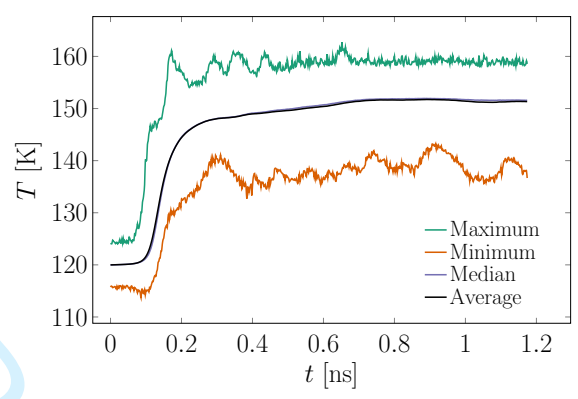

(b)

Figure 10: Evolution of maximum, minimum, median and average values of normalized number density as well as temperature in the whole simulation domain for the test case (A). Results are obtained using the DFP-SP model.

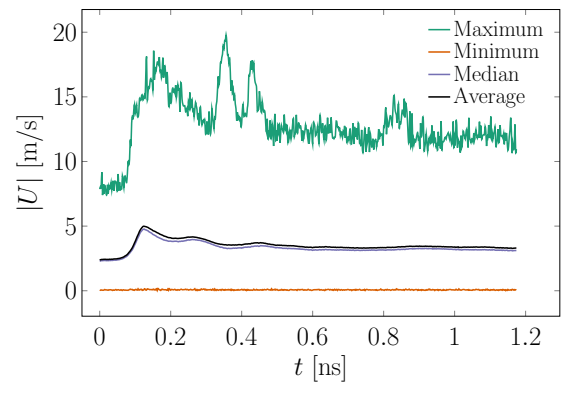

(a)

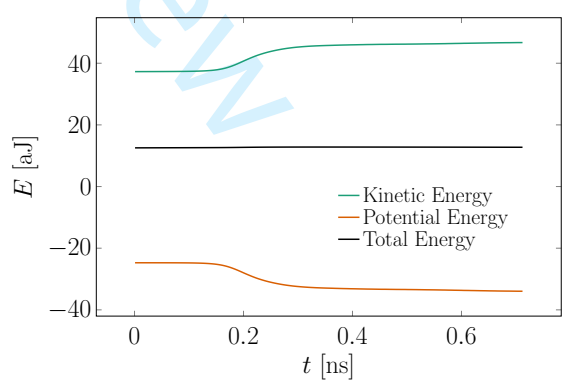

(b)

Figure 11: Evolution of maximum, minimum, median and average values of velocity magnitude in the whole simulation domain for the test case (A) shown in (a). The evolution of kinetic, potential and total energy of the system are depicted in (b). Here, [a] denotes the prefix unit called atto where $\mathrm{a}=10^{-18}$. Results are obtained using the DFP-SP model. 


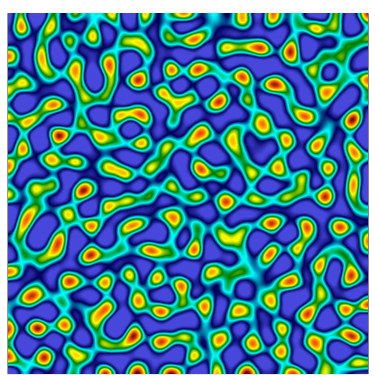

$t=0.066 \mathrm{~ns}$

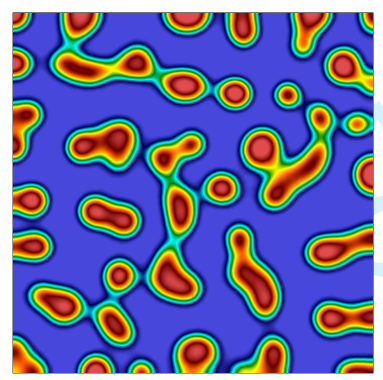

$t=0.294 \mathrm{~ns}$
Phase transition of droplets

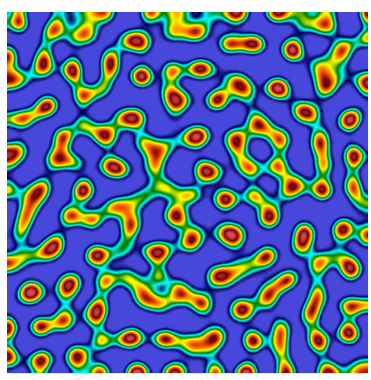

$t=0.108 \mathrm{~ns}$

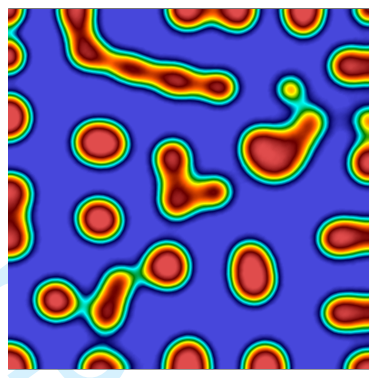

$t=0.546 \mathrm{~ns}$

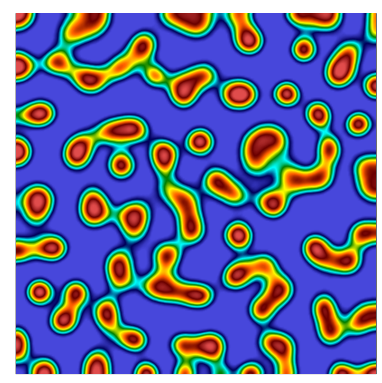

$t=0.192 \mathrm{~ns}$

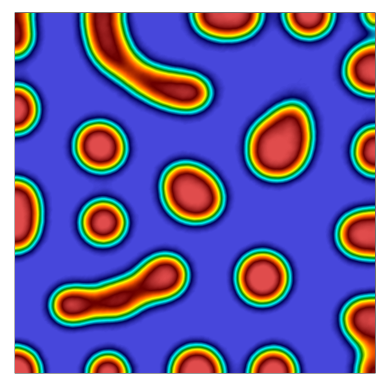

$t=0.876 \mathrm{~ns}$

Figure 12: Evolution of normalized number density in the spinodal decomposition of Argon obtained from simulation results of the DFP-SP model for test case (B), with initial number density $n^{(0)}=5 \times 10^{27} \mathrm{~m}^{-3}$ and temperature $T^{(0)}=120 \mathrm{~K}$ in the domain $\Omega_{x}=[0, L]^{2} \times[0,1] \mathrm{m}^{3}$ where $L=5 \times 10^{-8} \mathrm{~m}$.

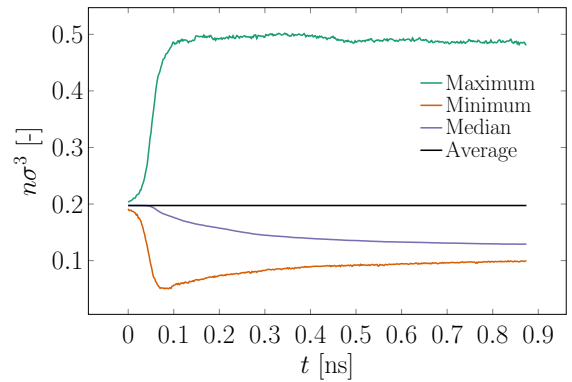

(a)

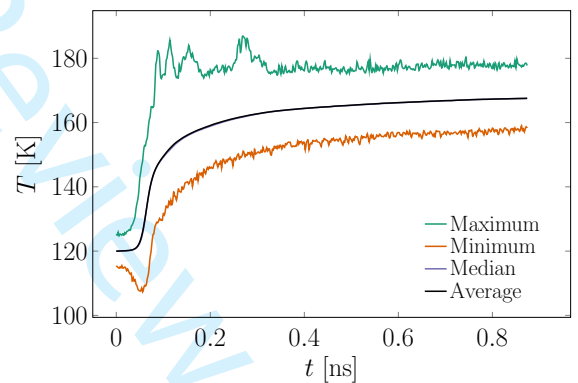

(b)

Figure 13: Evolution of maximum, minimum, median and average values of normalized number density as well as temperature in the whole simulation domain $\Omega_{x}$ for spinodal decomposition of Argon with settings of case (B). Results are obtained using the DFP-SP model.

combination with a fast elliptic solver. First the accuracy and performance of the devised DFP-SP model are assessed in predicting the surface tension and the evaporation rate of a liquid slab once in contact with the vacuum and once in contact with the equilibrium vapor phase. Furthermore, two intriguing multi-phase problems were addressed. The inverted-temperature gradient phenomenon which is observed in the vapor phase contained between two liquid slabs kept at slightly different temperatures, was accurately reproduced by the DFP-SP model. Further insights into the phenomenon were gained by analyzing single droplet evaporation process. Next, the spinodal decomposition of Argon 


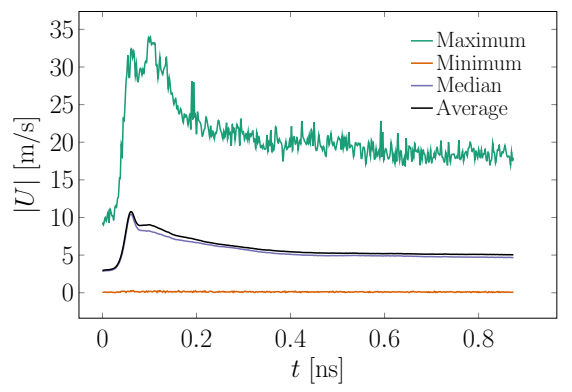

(a)

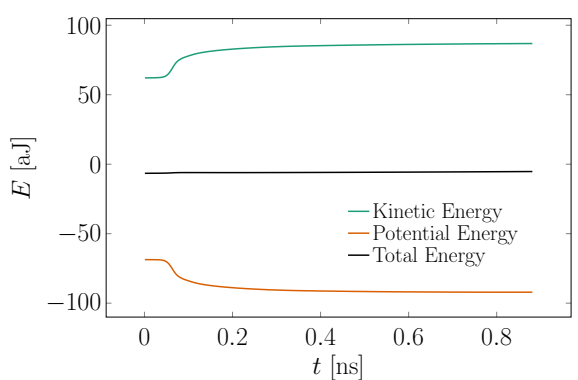

(b)

Figure 14: Evolution of maximum, minimum, median and average values of velocity magnitude in the whole domain $\Omega_{x}$ for the test case (B) in simulating the spinodal decomposition using the DFP-SP model are shown in (a). The evolution of kinetic, potential and total energy of the system are depicted in (b).

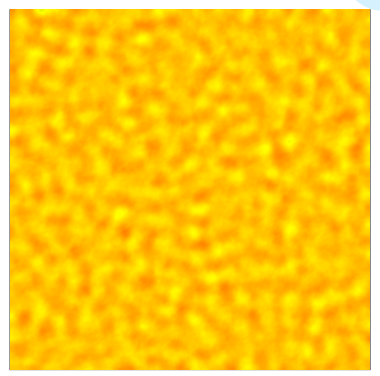

$t=0.02 \mathrm{~ns}$

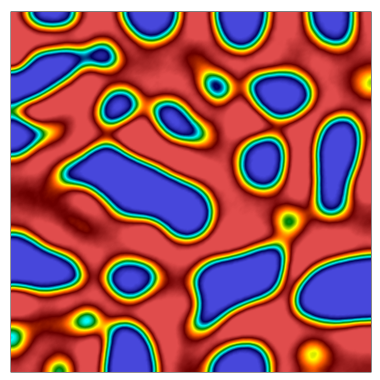

$t=0.245 \mathrm{~ns}$

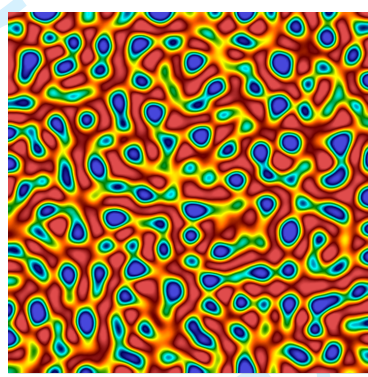

$t=0.056 \mathrm{~ns}$

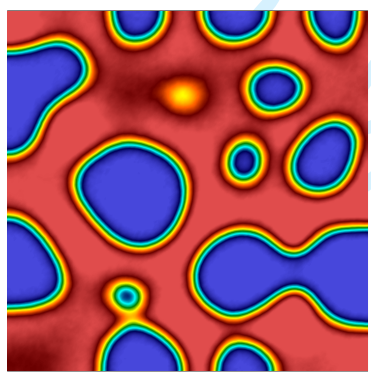

$t=0.646 \mathrm{~ns}$

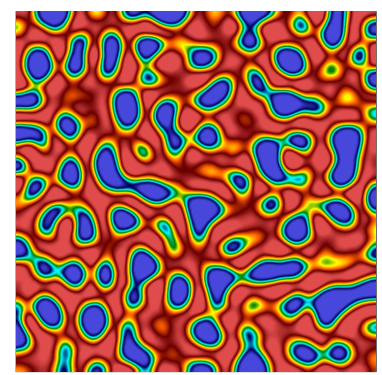

$t=0.09 \mathrm{~ns}$

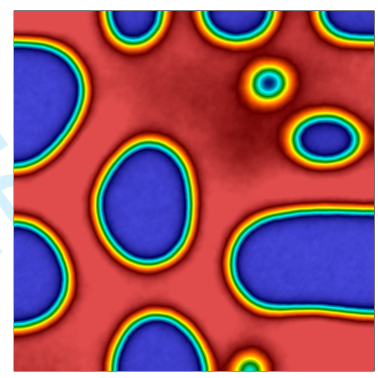

$t=1.304 \mathrm{~ns}$

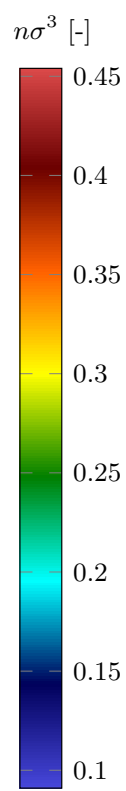

0.1

Figure 15: Evolution of normalized number density in the spinodal decomposition of Argon obtained from simulation results of the DFP-SP model for test case (C), with initial number density $n^{(0)}=8 \times 10^{27} \mathrm{~m}^{-3}$ and temperature $T^{(0)}=120 \mathrm{~K}$ in the domain $\Omega_{x}=[0, L]^{2} \times[0,1] \mathrm{m}^{3}$ where $L=5 \times 10^{-8} \mathrm{~m}$.

was investigated at different initial densities. Formation and coalescence of bubbles as well as droplets are illustrated. Moreover, the droplet growth rates are found to be consistent with the Lifschitz-Slyozov solution. Future steps will focus on integration of more accurate molecular potentials such as the Lennard-Jones potential into the Fokker-Planck-Poisson framework. We expect that this can be achieved by employing a system of SP equations. Moreover, extension of the model towards polyatomic degrees-of-freedom, mixtures and chemical reactions will be pursued. 


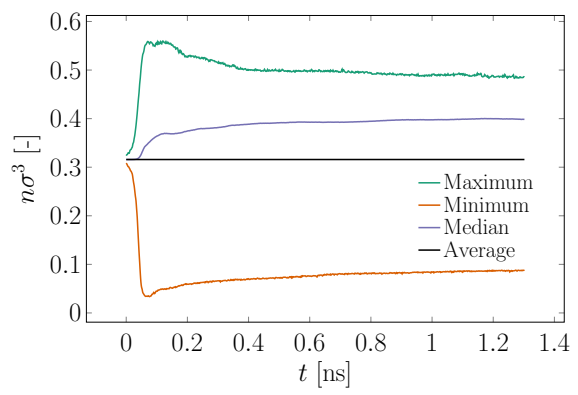

(a)

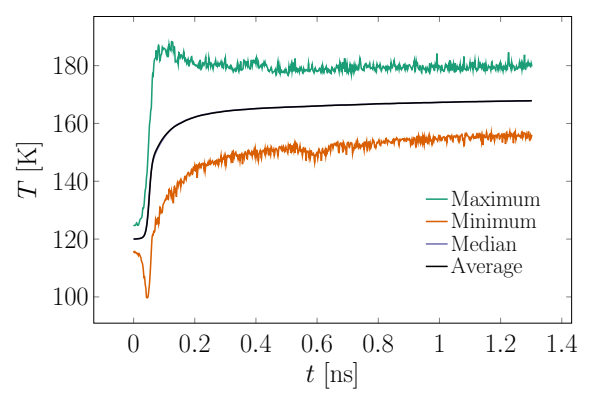

(b)

Figure 16: Evolution of maximum, minimum, median and average values of normalized number density as well as temperature in the whole simulation domain $\Omega_{x}$ for spinodal decomposition of Argon with settings of case (C). Results are obtained using the DFP-SP model.

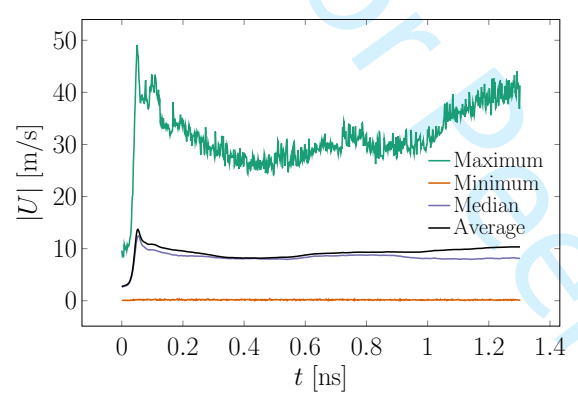

(a)

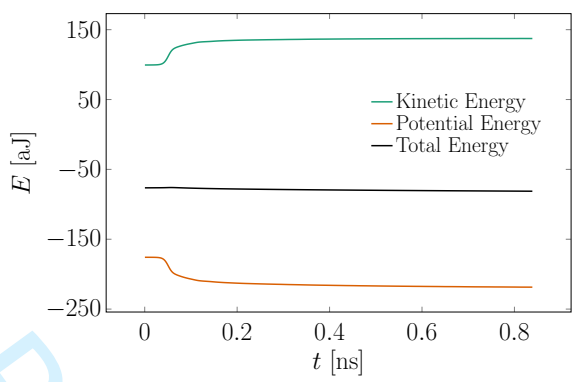

(b)

Figure 17: Evolution of maximum, minimum, median and average values of velocity magnitude in the whole domain $\Omega_{x}$ for the test case (C) in simulating spinodal decomposition using the DFP-SP model are shown in (a). The evolution of kinetic, potential and total energy of the system are depicted in (b).

\section{Acknowledgements}

The authors gratefully acknowledge the supports of Manuel Torrilhon throughout this study. Furthermore, we appreciate the stimulating discussions with Patrick Jenny. Mohsen Sadr acknowledges the financial support provided by Deutscher Akademischer Austauschdienst with grant number 57438025. Hossein Gorji acknowledges the funding provided by the Swiss National Science Foundation under grant number 174060. Marcel Pfeiffer gratefully acknowledge the Deutsche Forschungsgemeinschaft (DFG) for funding this research within the projects "Partikelverfahren mit Strahlungslser zur Simulation hochenthalper Nichtgleichgewichts-Plasmen" (project number 393159129).

\section{Appendix A. ESMC-Vlasov solution algorithm}

Here first the Monte-Carlo approach to account for the Enskog equation i.e. ESMC is reviewed. Then, the direct approach in solving the Vlasov integral is explained. At the end, the combined ESMC-Vlasov algorithm is presented which is deployed in this work as the benchmark solution of the EV equation. For more details on DSMC approach for 

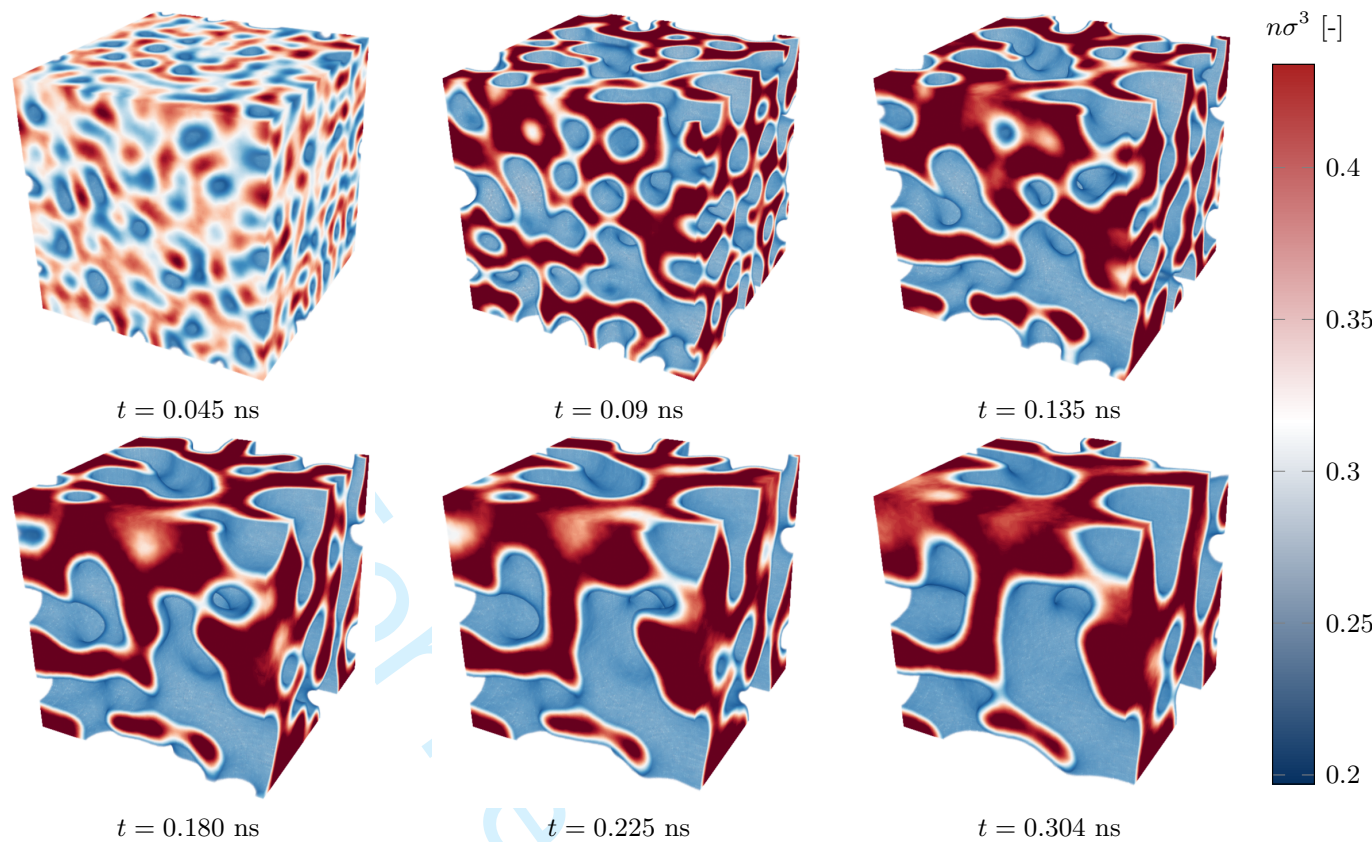

Figure 18: Evolution of normalized number density for the spinodal decomposition of Argon in three-dimensional physical space $\Omega_{x}=[0, L]^{3} \mathrm{~m}^{3}$ where $L=3 \times 10^{-8} \mathrm{~m}$ obtained from simulation results of the DFP-SP model, with initial number density $n^{(0)}=$ $8 \times 10^{27} \mathrm{~m}^{-3}$ and temperature $T^{(0)}=120 \mathrm{~K}$.

flow simulation based on the EV equation see Frezzotti et al. (2019).

Consider the position $X$ and the velocity $V$ as random variables which describe the evolution of $\mathcal{F}$ in the EV equation. As a Monte-Carlo particle method, instead of solving for $\mathcal{F}$ directly, the samples of position and velocity are evolved. Hence, at each time step first the velocity of particles are evolved following a jump process. Then, particles with new velocities are streamed freely till the end of time step.

\section{A.1. ESMC collision step}

The modified ESMC solution algorithm was introduced to mimic the solution algorithm of classical Bird's DSMC method with the modification for collision frequency as well as incorporating the fact that the colliding pair might lie in different cells. Using Bird's No-Time-Counter (NTC) approach (Bird 1994), particles collide with the probability of

$$
\mathcal{P}_{\text {coll. }}=\frac{\sigma_{T} c_{r} Y\left(n_{\text {mid }}\right)}{\left\{\sigma_{T} c_{r} Y\left(n_{\text {mid }}\right)\right\}_{\max }}
$$

where $\sigma_{T}=\pi \sigma^{2}$ is the hard-sphere collision cross-section, $g_{r}$ the magnitude of relative velocity for a colliding pair with number density of $n_{\text {mid }}$ at their midpoint. Here, the subscript max indicates an upper limit for the values of $\sigma_{T} c_{r}$ in the the computational cell. Particle pair with the index $(i, j)$ collides while satisfying the conservation laws. 
Bringing the randomness in the post-collision direction of velocity, the velocity of the colliding pair during a collision evolves as

$$
\begin{aligned}
V^{(i)} & =V_{\mathrm{c}}+\frac{g}{2} \text { and } \\
V^{(j)} & =V_{\mathrm{c}}-\frac{g}{2}, \quad \text { where } \\
g & =g_{r}[\cos (\theta), \sin (\theta) \cos (\phi), \sin (\theta) \sin (\phi)]^{T} .
\end{aligned}
$$

Consistent with the hard-sphere model, the angles of the post-collision relative velocity $g$ are drawn randomly with the uniform probability on the surface of the ball $\mathcal{B}(0,1)$ which is centered at zero and has the unit radius. Due to efficiency in sampling from one dimensional uniform distribution in practice, the angles $\theta$ and $\phi$ can be easily sampled using the map

$$
\begin{aligned}
& \theta=\arccos \left(2 \alpha_{1}-1\right) \text { and } \\
& \phi=2 \pi \alpha_{2},
\end{aligned}
$$

where $\alpha_{1,2} \sim \mathcal{U} \mathcal{R}([0,1])$, i.e. continuous uniform distribution with support in the interval $[0,1]$. The velocity of center of mass $V_{\mathrm{c}}:=\left(V^{(i)}+V^{(j)}\right) / 2$ and the magnitude of relative velocity $g:=V^{(i)}-V^{(j)}$, i.e. $g_{r}:=|g|$, remain constant during elastic collisions. Let $\hat{k}$ indicate the unit vector with the direction pointing to the center of $j$ th particle from $i$ th particle's center of mass. Hence, the number of collision candidates $N_{\text {cand }}^{(I)}$ for the time step size $\Delta t$ in the cell with index $I$ and $N_{p}^{(I)}$ particles is

$$
\begin{aligned}
N_{\text {cand }}^{(I)} & =\frac{1}{2} \omega_{\max }^{(I)} N_{p}^{(I)}, \text { where } \\
w_{\max }^{(I)} & =4 \pi \sigma^{2} \max _{(i j)}\left\{(g, k) n^{(J)} Y\left(n_{\text {mid }}\right)\right\} \Delta t .
\end{aligned}
$$

We initialize the upper bound of the collision rate using the thermal velocity

$$
\begin{aligned}
\omega_{\max , 0}^{(I)} & =4 \pi \sigma^{2} n^{(I)} \Theta_{0}^{(I)} Y\left(n^{(I)}\right) \Delta t, \quad \text { where } \\
\Theta_{0}^{(I)} & =10 \sqrt{\frac{k T_{0}^{(I)}}{m}}
\end{aligned}
$$

and update it every time step if a collision with $\omega^{(i j)}>w_{\max }^{(I)}$ where

$$
\omega^{(i j)}:=4 \pi \sigma^{2}(g, \hat{k}) n^{(J)} Y\left(n_{\text {mid }}\right) \Delta t
$$

occurs. Details of the implemented solution algorithm is described in Alg. 2. Note that here, $\mathcal{U I}([a, b])$ for $a, b \in \mathbb{N}$ and $b \geqslant a$ indicates a uniform discrete distribution over the set of natural numbers between $a$ and $b$. Furthermore, simply looping over cells and performing Enskog collisions similar to the implementation of DSMC may lead to introducing bias due to outside cell collisions and the fact that particles of one cell always are considered for collision before another. Therefore, an unbiased particle selection algorithm is devised here for the ESMC algorithm.

\section{A.2. Numerics of long-range interaction}

Similar to the SP model, first the Vlasov integral is estimated numerically on the faces of the computational cell indexed $(i, j, k)$. It is simpler to take the integral in spherical coordinates and read the density of the quadrature points from the density field. Then, 


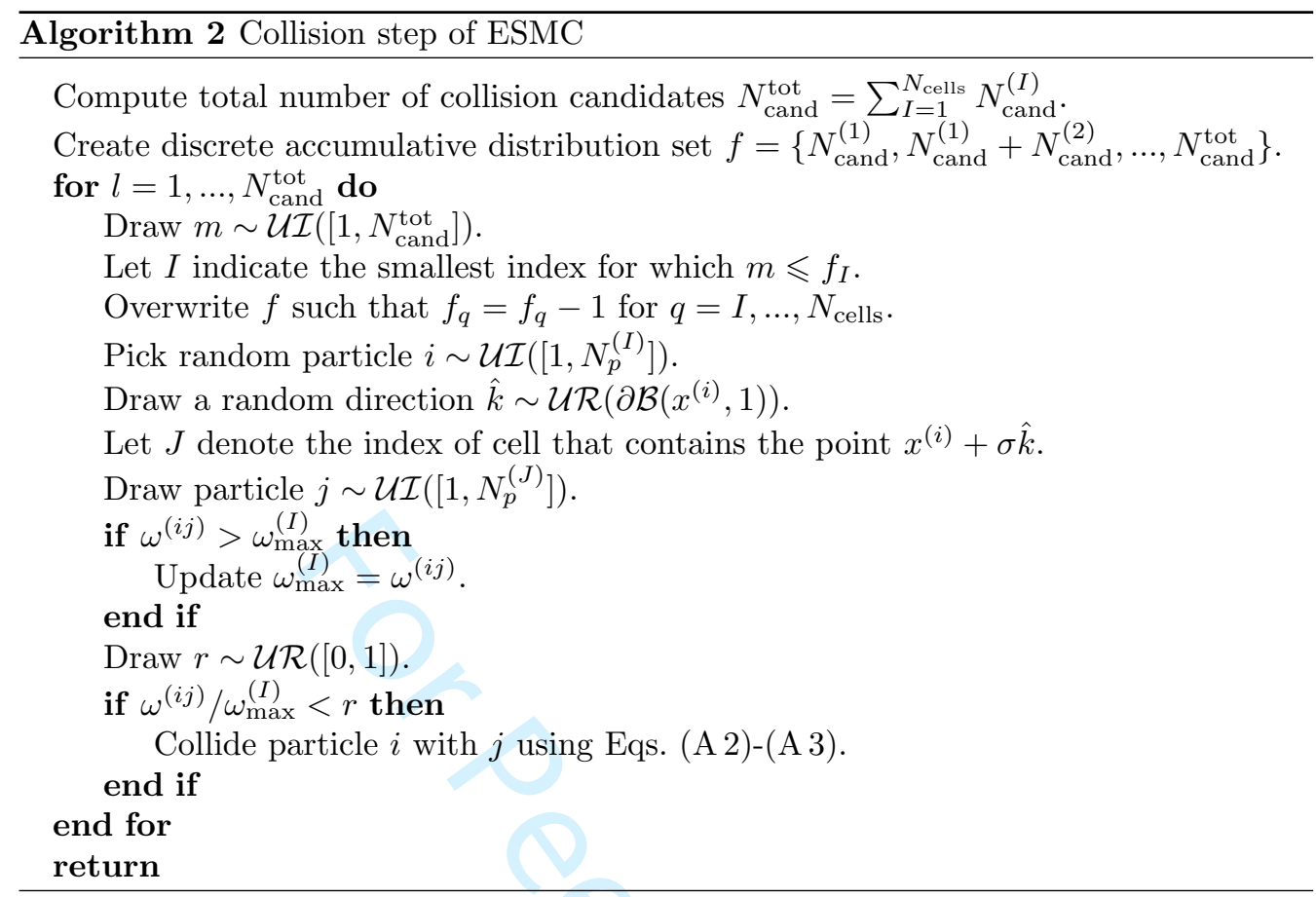

the numerical estimate of the Vlasov integral with origin at the center of the face that is indexed by $f_{l}$ becomes

$$
\begin{aligned}
& U_{f_{l}^{(i, j, k)}}^{(n)}=\phi_{0} \sum_{\alpha=1}^{N_{r}} \sum_{\beta=1}^{N_{\phi}} \sum_{\gamma=1}^{N_{\theta}}\left(\frac{\sigma}{r^{(\alpha)}}\right)^{6} n\left(y^{(\alpha, \beta, \gamma)}\right)\left(r^{(\alpha)}\right)^{2} \sin \left(\phi^{(\beta)}\right) \Delta r^{(\alpha)} \Delta \phi^{(\beta)} \Delta \theta^{(\gamma)}, \\
& y^{(\alpha, \beta, \gamma)}=\left(r^{(\alpha)} \cos \left(\theta^{(\gamma)}\right) \sin \left(\phi^{(\beta)}\right), r^{(\alpha)} \sin \left(\theta^{(\gamma)}\right) \sin \left(\phi^{(\beta)}\right), r^{(\alpha)} \cos \left(\phi^{(\beta)}\right)\right)^{T} .
\end{aligned}
$$

Let us combine the variables in a vector $x=[r, \phi, \theta]^{T}$ with lower and upper bounds in vectors $a=[\sigma, 0,0]^{T}$ and $b=\left[r_{\text {cut }}, \pi, 2 \pi\right]^{T}$, respectively. For numerical integration, Legendre-Gauss quadrature rule using $N_{r}=N_{\phi}=N_{\theta}=10$ are employed here. The $\eta$ 'th quadrature weight $w$ and point $\xi$ from the standard interval $[-1,1]$ are simply mapped back to the interval of interest for the $s$ 'th component of $x$ via

$$
\left\{\begin{array}{l}
x_{s}^{(\eta)}=\xi^{(\eta)} \frac{b_{s}-a_{s}}{2}+\frac{a_{s}+b_{s}}{2} \text { and } \\
\Delta x_{s}^{(\eta)}=w^{(\eta)} \frac{b_{s}-a_{s}}{2} .
\end{array}\right.
$$

Once the potential on the faces are computed numerically, the attractive forces are estimated via finite differences.

\section{A.3. ESMC-Vlasov algorithm}

To combine long-range interaction along with the short-range one, first at each time step, the ESMC collision step is carried out. Then, the velocities are updated to include attractive contribution once the numerical integration of the Vlasov on the faces are evaluated. Algorithm 3 shows the outline of the the ESMC-Vlasov solution algorithm used as the benchmark in this study. 


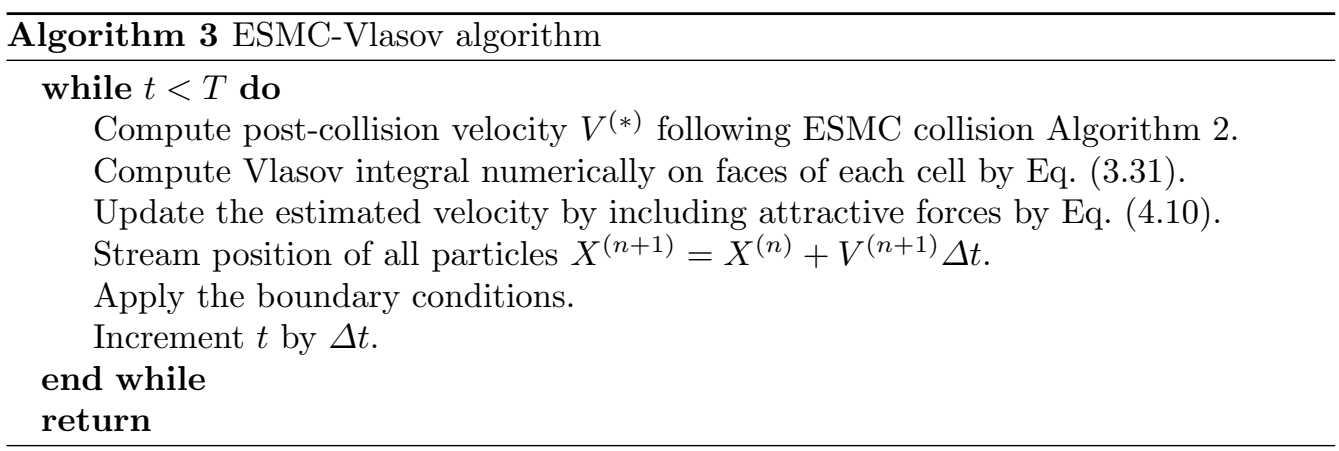

\section{Appendix B. Hybridized Discontinuous Galerkin Solver}

The solver used for the screened Poisson equation in the parallel high performance computing (HPC) case is based on the hybridized Discontinuous Galerkin solver in the particle code PICLas (Fasoulas et al. 2019). This solver uses a spectral element representation and is therefore referred to as hybridized Discontinuous Galerkin spectral element method (HDGSEM). The main advantage of this method is its reasonably high performance in parallelization. Here, an overview and introduction of the concept is given. The detailed derivation of the method as well as its application in simulations of plasma physics can be found in Pfeiffer et al. (2019).

As a first step, the screened Poisson equation (4.8) is decomposed into a first order system of partial differential equations, i.e.,

$$
\lambda^{2} u-\frac{\partial q_{i}}{\partial x_{i}}=n, \quad q_{i}=\frac{\partial u}{\partial x_{i}},
$$

with Dirichlet and Neumann boundary conditions encoded by

$$
\left.u\right|_{\partial \Omega_{\mathrm{Dir}}}=u_{\mathrm{Dir}},\left.\quad q_{i} \tilde{n}_{i}\right|_{\partial \Omega_{\mathrm{Neu}}}=\left(q_{i} \tilde{n}_{i}\right)_{\mathrm{Neu}} .
$$

Here, $\tilde{n}$ denotes outward unit normal vector on the boundary surface of the domain. One can obtain the variational form by multiplying the equations with test functions $\bar{u}$ and $\bar{q}$, respectively, and integrate over the volume of each element $K$, i.e.,

$$
\begin{aligned}
\left\langle\lambda^{2} u, \bar{u}\right\rangle_{K}-\left\langle\frac{\partial q_{i}}{\partial x_{i}}, \bar{u}\right\rangle_{K} & =\langle n, \bar{u}\rangle_{K} \\
\left\langle q_{i}, \bar{q}_{i}\right\rangle_{K} & =\left\langle\frac{\partial u}{\partial x_{i}}, \bar{q}_{i}\right\rangle_{K} .
\end{aligned}
$$

Here, $\left\langle a_{i}, b_{i}\right\rangle_{K}$ denotes the integration of the inner-product $a_{i} b_{i}$ inside the K'th element. Next, these equations are integrated by parts and discontinuous solutions at element interfaces are allowed for $q$ by applying jump condition. The main idea of the HDG method is to introduce an additional unknown $\theta$, which is unique on each element interface, in order to reduce dimension of the global system to the number of interfaces. By skipping several steps in the derivation, the final system of equations including the auxiliary variable $\theta$ as well as stabilization parameter $\tau>0$, see Cockburn et al. (2009), 
can be obtained as

$$
\begin{aligned}
\left\langle\lambda^{2} u, \bar{u}\right\rangle_{K}+\left\langle\frac{\partial q_{i}}{\partial x_{i}}, \bar{u}\right\rangle_{K}-\sum_{e \in \partial K}\{\tau u \bar{u}\}_{e}+\sum_{e \in \partial K}\{\tau \theta \bar{u}\}_{e} & =\langle n, \bar{u}\rangle_{K} \\
\left\langle q_{i}, \bar{q}_{i}\right\rangle_{K}+\left\langle u, \frac{\partial \bar{q}_{i}}{\partial x_{i}}\right\rangle_{K}-\sum_{e \in \partial K}\left\{\theta \bar{q}_{i} \tilde{n}_{i}\right\}_{e} & =0 \\
\text { and } \sum_{e \in \partial K, K^{\prime}}\left(\left\{q_{i} \tilde{n}_{i} \bar{\theta}\right\}_{e}+\{\tau u \bar{\theta}\}_{e}-\{\tau \theta \bar{\theta}\}_{e}\right) & =\sum_{e \in \Omega_{\mathrm{Neu}}}\left\{q_{i} \tilde{n}_{i} \bar{\theta}\right\}_{e},
\end{aligned}
$$

with the adjacent element $K^{\prime}$ on the interface indicated by $e$. Note that here $\{s\}_{e}$ indicates average of $s$ on the edge $e$, see Arnold et al. (2002). For the sake of simplicity, the transformation to/from the reference space is not explained here. This transformation as well as a detailed derivation of Eqs. (B 5)-(B 7) can be found in Pfeiffer et al. (2019).

In order to find the solution $u$ of Eq. (B 1), first, a global system of equations is solved for the auxiliary variable $\theta$ only on the surfaces using a conjugate-gradient method. The final volume solution $u$ inside each cell is calculated in a post processing step locally in each element. Once the solution $u$ with expansion in polynomial basis functions inside each cell is found, its derivatives needed for computing attractive force $H$ at any point inside the cell can be obtained simply by applying derivatives on the basis functions.

\section{Appendix C. Total Energy for Enskog-Vlasov System}

In order to derive evolution of the total energy in the EV equation, let us first multiply Eq. (2.3) by $v_{j} v_{j} / 2$ and integrate over $v$. Therefore we get

$$
\frac{\partial}{\partial t} \int_{\mathbb{R}^{3}} \frac{1}{2} \mathcal{F} v_{j} v_{j} d v+\frac{\partial}{\partial x_{i}}\left(\frac{1}{2} \int_{\mathbb{R}^{3}} v_{i} v_{j} v_{j} \mathcal{F} d v\right)-\int_{\mathbb{R}^{3}} v_{j} \frac{H_{j}}{m} \mathcal{F} d v=0 .
$$

Using the notation

$$
\mathbb{E}[Q(V \mid x, t)]=\frac{1}{\rho} \int_{\mathbb{R}^{3}} Q(v) \mathcal{F}(v, x, t) d v
$$

yields

$$
\frac{\partial}{\partial t}\left(\frac{1}{2} \rho \mathbb{E}\left[V_{j} V_{j} \mid x, t\right]\right)+\frac{\partial}{\partial x_{i}}\left(\frac{1}{2} \rho \mathbb{E}\left[V_{i} V_{j} V_{j} \mid x, t\right]\right)-\frac{H_{j}}{m} \rho \mathbb{E}\left[V_{j} \mid x, t\right]=0 .
$$

Now by taking integral over the physical space $\Omega$ we get

$$
\begin{aligned}
& \frac{\partial}{\partial t} \int_{\Omega}\left(\frac{1}{2} \rho \mathbb{E}\left[V_{j} V_{j} \mid x, t\right] d x\right)+\int_{\partial \Omega}\left(\frac{1}{2} \rho \mathbb{E}\left[V_{i} V_{j} V_{j} \mid x, t\right] n_{i} d s\right) \\
&+\int_{\partial \Omega}\left(\frac{\Phi}{m} \rho \mathbb{E}\left[V_{j} \mid x, t\right] n_{j} d s\right)+\int_{\Omega} \frac{\Phi}{m} \frac{\partial \rho}{\partial t} d x=0,
\end{aligned}
$$

where $\partial \Omega$ is the boundary of $\Omega$ and $n$ its normal. Therefore for the interval $t=0$ to $t=t_{f}$ we get

$$
\begin{aligned}
E_{\mathrm{kin}}\left(t_{f}\right)= & E_{\mathrm{kin}}(0)-\int_{0}^{t_{f}} \int_{\Omega} \frac{\Phi}{m} \frac{\partial \rho}{\partial t} d x d t \\
& -\int_{0}^{t_{f}} \int_{\substack{\partial \Omega \\
\text { Cambridge University Press }}}\left(\frac{1}{2} \mathbb{E}\left[V_{i} V_{j} V_{j} \mid x, t\right]+\frac{\Phi}{m} \mathbb{E}\left[V_{i} \mid x, t\right]\right) \rho n_{i} d s d t,
\end{aligned}
$$


where $E_{\text {kin }}=\int_{\Omega} 1 / 2 \rho \mathbb{E}\left[V_{j} V_{j} \mid x, t\right] d x$. The surface term can be computed by averaging over particles crossing the boundary of the domain. If we have the homogeneous boundary condition however, it simplified to

$$
E_{\mathrm{kin}}\left(t_{f}\right)=E_{\mathrm{kin}}(0)-\int_{0}^{t_{f}} \int_{\Omega} \frac{\Phi}{m} \frac{\partial \rho}{\partial t} d x d t .
$$

Since $\Phi$ depends on $\rho$ through a time independent symmetric kernel

$$
\Phi(x, t)=\int_{\Omega} \mathcal{K}\left(x^{\prime}, x\right) \rho\left(x^{\prime}, t\right) d x^{\prime},
$$

we obtain

$$
\frac{\partial \Phi}{\partial t}=\int_{\Omega} \mathcal{K} \frac{\partial \rho}{\partial t} d x
$$

and thus

$$
\begin{aligned}
\int_{\Omega} \rho \frac{\partial \Phi}{\partial t} d x & =\int_{\Omega} \rho(x) \int_{\Omega} \mathcal{K}\left(x^{\prime}, x\right) \frac{\partial \rho\left(x^{\prime}\right)}{\partial t} d x^{\prime} d x \\
& =\int_{\Omega} \frac{\partial \rho\left(x^{\prime}\right)}{\partial t} \int_{\Omega} \mathcal{K}\left(x^{\prime}, x\right) \rho(x) d x d x^{\prime} \\
& =\int_{\Omega} \frac{\partial \rho\left(x^{\prime}\right)}{\partial t} \int_{\Omega} \mathcal{K}\left(x, x^{\prime}\right) \rho(x) d x d x^{\prime} \\
& =\int_{\Omega} \frac{\partial \rho}{\partial t} \Phi d x^{\prime} .
\end{aligned}
$$

Therefore Eq. (C 6) results in the following energy conservation

$$
E_{\mathrm{kin}}\left(t_{f}\right)+E_{\mathrm{pot}}\left(t_{f}\right)=E_{\mathrm{kin}}(0)+E_{\mathrm{pot}}(0),
$$

where $E_{\text {pot }}=1 / 2 \int_{\Omega} \rho \Phi / m d x$ is the potential energy.

\section{REFERENCES}

Alexander, F. J., Garcia, A. L. \& Alder, B. J. 1995 A consistent Boltzmann algorithm. Physical Review Letters 74 (26), 5212.

Alharthy, Najeeb S, Nguyen, Thanh, Teklu, T, Kazemi, Hossein, Graves, R \& others 2013 Multiphase compositional modeling in small-scale pores of unconventional shale reservoirs. In SPE Annual Technical Conference and Exhibition. Society of Petroleum Engineers.

Arnold, Douglas N, Brezzi, Franco, Cockburn, Bernardo \& Marini, L Donatella 2002 Unified analysis of discontinuous galerkin methods for elliptic problems. SIAM journal on numerical analysis 39 (5), 1749-1779.

Bastea, S \& Lebowitz, JL 1997 Spinodal decomposition in binary gases. Physical review letters $\mathbf{7 8}(18), 3499$.

Benson, Craig M, Levin, Deborah A, Zhong, Jiaqiang, Gimelshein, Sergey F \& Montaser, Akbar 2004 Kinetic model for simulation of aerosol droplets in hightemperature environments. Journal of thermophysics and heat transfer 18 (1), 122-134.

BIRD, G. A. 1963 Approach to translational equilibrium in a rigid sphere gas. Physics of Fluids 6 (10), 1518-1519.

BIRD, G. A. 1994 Molecular gas dynamics and the direct simulation of gas flows. Clarendon Press.

Broadwell, J. E. 1964 Study of rarefied shear flow by the discrete velocity method. Journal of Fluid Mechanics 19 (3), 401-414.

Carnahan, Norman F \& Starling, Kenneth E 1969 Equation of state for nonattracting rigid spheres. The Journal of chemical physics $\mathbf{5 1}$ (2), 635-636. 
Cercignani, CARlo 1988a The Boltzmann equation. In The Boltzmann equation and its applications, pp. 40-103. Springer.

Cercignani, C. $1988 b$ The Boltzmann Equation and Its Applications. Springer.

Chapman, S. \& Cowling, T. G. 1970 The mathematical theory of non-uniform gases: an account of the kinetic theory of viscosity, thermal conduction and diffusion in gases. Cambridge university press.

Cockburn, Bernardo, Dong, Bo, Guzmán, Johnny, Restelli, Marco \& Sacco, RICCARDO 2009 A hybridizable discontinuous galerkin method for steady-state convectiondiffusion-reaction problems. SIAM Journal on Scientific Computing 31 (5), 3827-3846.

Enskog, D. 1922 Kinetische Theorie der Wärmeleitung: Reibung und Selbst-diffusion in Gewissen verdichteten gasen und flüssigkeiten. Almqvist \& Wiksells boktryckeri-a.-b.

Espanol, Pep \& Revenga, Mariano 2003 Smoothed dissipative particle dynamics. Physical Review E 67 (2), 026705.

Fasoulas, S., Munz, C.-D., Pfeiffer, M., Beyer, J., Binder, T., Copplestone, S., Mirza, A., Nizenkov, P., Ortwein, P. \& Reschke, W. 2019 Combining particle-incell and direct simulation monte carlo for the simulation of reactive plasma flows. Physics of Fluids 31 (7), 072006.

Frezzotti, A. 1997 A particle scheme for the numerical solution of the Enskog equation. Physics of Fluids 9 (5), 1329-1335.

Frezzotti, A, Barbante, P \& Gibelli, L 2019 Direct simulation monte carlo applications to liquid-vapor flows. Physics of Fluids 31 (6), 062103.

Frezzotti, A, Gibelli, L, Lockerby, DA \& Sprittles, JE 2018 Mean-field kinetic theory approach to evaporation of a binary liquid into vacuum. Physical Review Fluids 3 (5), 054001 .

Frezzotti, Aldo, Gibelli, Livio \& Lorenzani, Silvia 2005 Mean field kinetic theory description of evaporation of a fluid into vacuum. Physics of Fluids 17 (1), 012102.

Frezzotti, Aldo, Grosfils, Patrick \& Toxvaerd, Soeren 2003 Evidence of an inverted temperature gradient during evaporation/condensation of a lennard-jones fluid. Physics of fluids 15 (10), 2837-2842.

Gamba, Irene M \& Tharkabhushanam, Sri Harsha 2009 Spectral-lagrangian methods for collisional models of non-equilibrium statistical states. Journal of Computational Physics 228 (6), 2012-2036.

Ghoufi, Aziz \& Malfreyt, Patrice 2010 Calculation of the surface tension from multibody dissipative particle dynamics and monte carlo methods. Physical Review E 82 (1), 016706.

GorJI, M. H. 2014 Fokker-Planck solution algorithm for rarefied gas flows and applications of complex gas-surface interactions. PhD thesis, Swiss Federal Institute of Technology in Zurich (ETHZ).

Gorji, M. Hossein \& Jenny, PAtrick 2013 A Fokker-Planck based kinetic model for diatomic rarefied gas flows. Physics of fluids 25 (6), 062002.

GorJi, M. H. \& Jenny, P. 2014 An efficient particle Fokker-Planck algorithm for rarefied gas flows. Journal of Computational Physics 262, 325-343.

Gorji, M. H., Torrilhon, M. \& Jenny, P. 2011 Fokker-Planck model for computational studies of monatomic rarefied gas flows. Journal of Fluid Mechanics 680, 574-601.

Grmela, M 1971 Kinetic equation approach to phase transitions. Journal of Statistical Physics $3(3), 347-364$.

He, Xiaoyi \& Doolen, Gary D 2002 Thermodynamic foundations of kinetic theory and lattice Boltzmann models for multiphase flows. Journal of Statistical Physics 107 (1-2), 309-328.

Heylmun, Jeffrey C, Kong, Bo, Passalacqua, Alberto \& Fox, Rodney O 2019 A quadrature-based moment method for polydisperse bubbly flows. Computer Physics Communications 244, 187-204.

Hirschfelder, J., Bird, R. B. \& Curtiss, C. F. 1964 Molecular theory of gases and liquids. Wiley.

Hudgins, Duane, Gambino, Nadia, Rollinger, Bob \& Abhari, Reza 2016 Neutral cluster debris dynamics in droplet-based laser-produced plasma sources. Journal of Physics D: Applied Physics 49 (18), 185205.

JENNy, PATRICK \& GoRJI, M. Hossein 2019 Accurate particle time integration for Cambridge University Press 
solving Vlasov-Fokker-Planck equations with specified electromagnetic fields. Journal of Computational Physics 387, 430-445.

Jenny, P., Torrilhon, M. \& Heinz, S. 2010 A solution algorithm for the fluid dynamic equations based on a stochastic model for molecular motion. Journal of Computational Physics 229 (4), 1077-1098.

Jun, Eunji, Pfeiffer, Marcel, Mieussens, Luc \& Gorji, M. Hossein 2019 Comparative study between cubic and ellipsoidal Fokker-Planck kinetic models. AIAA Journal $5 \mathbf{7}$ (6), $2524-2533$.

KARKheCK, John \& Stell, George 1981 Kinetic mean-field theories. The Journal of Chemical Physics 75 (3), 1475-1487.

Kobayashi, Kazumichi, Hori, Kazumasa, Kon, Misaki, Sasaki, Kiyofumi \& Watanabe, MASAO 2016 Molecular dynamics study on evaporation and reflection of monatomic molecules to construct kinetic boundary condition in vapor-liquid equilibria. Heat and Mass Transfer $\mathbf{5 2}$ (9), 1851-1859.

Kon, Misaki, Kobayashi, Kazumichi \& Watanabe, Masao 2014 Method of determining kinetic boundary conditions in net evaporation/condensation. Physics of Fluids 26 (7), 072003.

Korteweg, Diederick Johannes 1901 Sur la forme que prennent les équations du mouvements des fluides si l'on tient compte des forces capillaires causées par des variations de densité considérables mais connues et sur la théorie de la capillarité dans l'hypothèse d'une variation continue de la densité. Archives Néerlandaises des Sciences exactes et naturelles 6, $1-24$.

Kremer, GM \& Rosa JR, E 1988 On Enskog's dense gas theory. i. the method of moments for monatomic gases. The Journal of chemical physics 89 (5), 3240-3247.

Landau, Lev Davidovich \& Lifshitz, Evgeny Mikhailovich 1959 Fluid mechanics. flme .

Liu, Moubin, Meakin, Paul \& Huang, Hai 2007 Dissipative particle dynamics simulation of multiphase fluid flow in microchannels and microchannel networks. Physics of Fluids 19 (3), 033302.

Meland, RoAR 2003 Molecular dynamics simulation of the inverted temperature gradient phenomenon. Physics of Fluids 15 (10), 3244-3247.

Meland, Roar \& YTrehus, Tor 2004 Dependence of the inverted temperature gradient phenomenon on the condensation coefficient. Physics of Fluids 16 (3), 836-838.

Montanero, J. M. \& Santos, A. 1997 a Simulation of the Enskog equation a la Bird. Physics of Fluids 9 (7), 2057-2060.

Montanero, J. M. \& Santos, A. $1997 b$ Viscometric effects in a dense hard-sphere fluid. Physica A: Statistical Mechanics and its Applications 240 (1-2), 229-238.

OKSENDAL, BERnt 2013 Stochastic differential equations: an introduction with applications. Springer Science \& Business Media.

PAO, Young-PING 1971 Application of kinetic theory to the problem of evaporation and condensation. The physics of Fluids 14 (2), 306-312.

Pfeiffer, M. \& GorJi, M. H. 2017 Adaptive particle-cell algorithm for Fokker-Planck based rarefied gas flow simulations. Computer Physics Communications 213, 1-8.

Pfeiffer, M, Hindenlang, F, Binder, T, Copplestone, SM, Munz, C-D \& Fasoulas, S 2019 A particle-in-cell solver based on a high-order hybridizable discontinuous galerkin spectral element method on unstructured curved meshes. Computer Methods in Applied Mechanics and Engineering 349, 149-166.

PIEchór, K 1994 Discrete velocity models of the Enskog-Vlasov equation. Transport Theory and Statistical Physics 23 (1-3), 39-74.

Resibois, Pierre 1978 H-theorem for the (modified) nonlinear enskog equation. Journal of Statistical Physics 19 (6), 593-609.

Risken, H. 1989 The Fokker-Planck Equation: Methods of solution and applications. Springer.

SAdr, Mohsen \& GorJi, M. Hossein 2017 A continuous stochastic model for non-equilibrium dense gases. Physics of Fluids 29 (12), 122007.

Sadr, Mohsen \& GorJi, M. Hossein 2019 Treatment of long-range interactions arising in the Enskog-Vlasov description of dense fluids. Journal of Computational Physics 378, $129-142$. 
Schwarzkopf, John D, Sommerfeld, Martin, Crowe, Clayton T \& Tsuji, Yutaka 2011 Multiphase flows with droplets and particles. CRC press.

Shan, Xiaowen \& Chen, Hudong 1993 Lattice Boltzmann model for simulating flows with multiple phases and components. Physical review E 47 (3), 1815.

SigGia, ERIC D 1979 Late stages of spinodal decomposition in binary mixtures. Physical review A 20 (2), 595.

Struchtrup, Henning \& Frezzotti, Aldo 2019 Grads 13 moments approximation for Enskog-Vlasov equation. In AIP Conference Proceedings, , vol. 2132, p. 120007. AIP Publishing LLC.

Torrilhon, Manuel 2016 Modeling nonequilibrium gas flow based on moment equations. Annual review of fluid mechanics 48, 429-458.

Van BeiJeren, Henk \& Ernst, Matthieu H 1973 The modified enskog equation. Physica 68 (3), 437-456.

Vlasov, AA 1978 Many-particle theory and its application to plasma. New York, Gordon and Breach.

Wang, Peng, Wu, Lei, Ho, Minh Tuan, Li, Jun, Li, Zhi-Hui \& Zhang, Yonghao 2020 The kinetic shakhov-enskog model for non-equilibrium flow of dense gases. Journal of Fluid Mechanics $\mathbf{8 8 3}$.

Watanabe, Hiroshi, Ito, Nobuyasu \& Hu, Chin-Kun 2012 Phase diagram and universality of the lennard-jones gas-liquid system. The Journal of chemical physics 136 (20), 204102.

WÖRNER, MARTIN 2012 Numerical modeling of multiphase flows in microfluidics and micro process engineering: a review of methods and applications. Microfluidics and nanofluidics $12(6), 841-886$.

Wu, Lei, Liu, Hainu, Reese, Jason M \& Zhang, Yonghao 2016 Non-equilibrium dynamics of dense gas under tight confinement. Journal of Fluid Mechanics 794, 252-266.

Wu, Lei, White, Craig, Scanlon, Thomas J, Reese, Jason M \& Zhang, Yonghao 2013 Deterministic numerical solutions of the Boltzmann equation using the fast spectral method. Journal of Computational Physics 250, 27-52.

Wu, Lei, Zhang, Yonghao \& Reese, Jason M 2015 Fast spectral solution of the generalized enskog equation for dense gases. Journal of Computational Physics 303, 66-79.

Yasuoka, Kenji, Matsumoto, Mitsuhiro \& Kataoka, Yosuke 1994 Evaporation and condensation at a liquid surface. i. argon. The Journal of chemical physics 101 (9), 79047911. 